\title{
Vers une théorie du pâtir communicationnel. Sensibiliser
} Habermas

\section{Towards a theory of communicational feeling. Sensetizing Habermas}

\section{Hacia una teoría del sufrimiento comunicacional - Sensibilizar a Habermas}

\section{Mathieu Berger}

Numéro 62, hiver 2017

Peirce et les sciences sociales. Une sociologie pragmaticiste?

URI : https://id.erudit.org/iderudit/1045615ar

DOI : https://doi.org/10.7202/1045615ar

Aller au sommaire du numéro

Éditeur(s)

Athéna éditions

ISSN

0831-1048 (imprimé)

1923-5771 (numérique)

Découvrir la revue

Citer cet article

Berger, M. (2017). Vers une théorie du pâtir communicationnel. Sensibiliser Habermas. Cahiers de recherche sociologique, (62), 69-108.

https://doi.org/10.7202/1045615ar
Résumé de l'article

Ce texte tente de poser les jalons d'une critique interne et reconstructrice de la Théorie de l'agir communicationnel. A travers la discussion de penseurs chers à Jürgen Habermas (C.S. Peirce, K. Bühler, G. H. Mead), l'auteur plaide pour une " sensibilisation » du modèle de rationalité développé par le francfortois, condition d'une meilleure capacité de description des situations et processus de communication, autrement dit, d'un plus grand réalisme de la théorie : réalisme sémiotique, par la prise en compte de l'instabilité de la signification dans les situations d'interlocution (Bühler) et de la multi-modalité de sémioses combinant le symbolique, l'indiciel et l'iconique, aspects de tiercéité, de secondéité et de priméité (Peirce) ; réalisme écologique, à travers une plus grande attention pour les situations concrètes, pour les corps qui soutiennent la communication, ainsi que pour les milieux qui l'accueillent et la reçoivent (Mead). 


\title{
Vers une théorie du pâtir communicationnel. Sensibiliser Habermas
}

\author{
Mathieu Berger
}

\begin{abstract}
Reason's access to the world [...] is mediated by a feeling body, whose testimony certifies our validity claims [...]. As long as reason stays within the rarefied chamber [of the Platonic domain], it can abstract from concrete situation and take a profitable leave of one's emotional investments, but as soon as knowers step into the world of uncertainty, they inexorably fall back on an auxiliary intelligence about things themselves that only noncognitive faculties can gather. Human intelligence is emotional just as emotions are intelligent, and this is so because we live in the world of indeterminacy that no rational faculty and theoretical rigor can expunge.
\end{abstract}

DMitri SHALIN ${ }^{1}$

\section{Habermas et les affects}

Dans sa Théorie de l'agir communicationnel (TAC) ${ }^{2}$, Jürgen Habermas a reconstruit un modèle de rationalité enraciné dans une "pragmatique universelle» de la communication ${ }^{3}$. Ces travaux ont depuis trouvé d'importants

1. Dimitri Shalin, «Critical Theory and The Pragmatist Challenge», American Journal of Sociology, vol. 98, $n^{\circ} 2$, 1991, p. 256.

2. Jürgen Habermas, Théorie de l'agir communicationnel. Tomes 1 et 2, Paris, Fayard, 1987 (1981); désormais TAC 1 et TAC 2.

3. Jürgen Habermas, «What is Universal Pragmatics?», On The Pragmatics of communication, Cambridge, MIT Press, 1998 (1976), p. 21-104. 
prolongements en théorie politique, à travers l'élaboration d'un modèle de démocratie fondé sur une éthique de la discussion. Euvre monumentale, la TAC était motivée par des enjeux auxquels on associe généralement l'approche pragmatiste en philosophie et en sciences sociales, comme la «mise en situation de la raison» et la nécessité de «surmonter le logocentrisme qui a marqué la tradition occidentale» ${ }^{4}$. Or, aux yeux de bien des commentateurs, les pragmatistes en premier, ces qualités revendiquées par Habermas semblent inappropriées pour caractériser son entreprise. Quel nom vient en effet immédiatement à l'esprit lorsqu'on évoque en théorie politique le logocentrisme et l'abstraction des situations de communication, sinon celui du théoricien francfortois? Théorie de l'agir communicationnel, qui reste une référence essentielle pour les théories de la démocratie, a été l'objet d'un grand nombre de commentaires et de critiques, qui ont vu Habermas défendre son modèle inlassablement ${ }^{5}$, pour parfois le retravailler à la marge. Nous nous limiterons ici à un aspect de la théorie de l'agir communicationnel maintes fois pointé par les critiques, mais qui a fait l'objet de peu d'attention ou de révision par le théoricien allemand: sa dimension sensible ${ }^{6}$.

Ici, il faut d'abord défendre Habermas contre les stéréotypes faisant de la TAC l'exemple d'un rationalisme froid, insensible, dans l'étude de la communication intersubjective. Les affects et les émotions sont importants chez Habermas, l'étude des «sentiments moraux» constituant le point de départ de son «éthique de la discussion ${ }^{7}$ ». Si Habermas ne nie nullement l'importance des affects pour la communication éthique, il est par contre possible de pointer chez lui une sous-estimation des affects dans la communication. Comme suggéré à l'instant, les sentiments moraux sont considérés comme le point de départ, comme ce qui déclenche la discussion; ils ne sont pas, audelà, considérés comme une dimension centrale de la discussion à suivre. À ce titre, ils intéressent peu Habermas pour ce qui relève, au sens strict, d'une théorie de la communication. Ainsi, par exemple, dans On The Pragmatics of Communication ${ }^{8}$, très utile compilation de vingt ans de travaux en théorie de

4. Jürgen Habermas, TAC 1, op. cit., quatrième de couverture.

5. Jürgen Habermas, «A Reply To My Critics», dans J. B. Thompson et D. Held (dir.), Habermas. Critical Debates, Cambridge, MIT Press, 1982; "Questions and Counter-questions», dans R. Bernstein (dir.), Habermas and Modernity, Cambridge, Polity Press, 1985; «A Reply», dans A. Honneth et H. Joas, Communicative Action. Essays on Jürgen Habermas's The Theory of Communicative Action, Cambridge, MIT Press, 1991, p. 214-264; «Concluding Remarks», dans M. Aboulafia, M. Bookman, C. Kemp (dir.), Habermas and Pragmatism, Londres/ New York, Routledge, 2002.

6. Différentes parties et versions de ce texte ont été présentées au Congrès de l'Association Internationale de Sociologie de langue française de Montréal de 2016, et lors d'invitations à l'École Polytechnique Fédérale de Lausanne (EPFL) et à l'Université de Lausanne (UniL). Merci à Jean-François Coté, Luca Pattaroni, Philippe Gonzalez et Laurence Kaufmann pour ces opportunités; à Joan Stavo-Debauge, Eva Debray et Laurence Kaufmann, ainsi qu'aux évaluateurs anonymes, pour leurs commentaires et suggestions.

7. Jürgen Habermas, Morale et communication, Paris, Cerf, 1991 (1983), p. 67.

8. Jürgen Habermas, On The Pragmatics of Communication, Cambridge, MIT Press, 1998. 
la communication (1976-1996), on retrouve sur les 433 pages de l'ouvrage à peine quatre occurrences du terme "emotion (al)»; et il n'évoque les termes «affect(ual)» qu'en passant.

\section{Un exemple: l'humiliation}

Cette limite est manifeste lorsqu'on considère le statut attribué aux affects par Habermas - et Peter Strawson dont il commente l'essai Freedom and Resentment ${ }^{9}$ - dans l'exemple suivant, qui concerne une situation d'humiliation:

Strawson prend pour point de départ une réaction affective dont le caractère impérieux permet parfaitement de rendre manifeste le contenu de réalité des expériences morales à quiconque, soit-il l'homme le plus endurci. Il part, en l'occurrence, de l'indignation avec laquelle nous réagissons aux humiliations. Si l'offense causée n'est pas réparée, d'une façon ou d'une autre, cette réaction sans équivoque persiste et se renforce en un ressentiment latent. Or ce sentiment révèle par sa persistance qu'il y a une dimension morale dans le fait de subir une humiliation ${ }^{10}$.

Strawson et Habermas prennent ainsi pour «point de départ» de leur réflexion éthique la «réaction affective» à l'humiliation, ici résumée à l'«indignation» et à son appel «impérieux» à une action visant la «réparation» de l'offense, à travers - comme Habermas le précise plus loin - «des excuses». L'humiliation, immédiatement rapportée à la tonalité affective de l'indignation, est traitée comme l'incident déclencheur d'une action communicative de dénonciation, de justification, d'argumentation, etc. Les conséquences affectives et pathiques de l'humiliation laissent ainsi bien vite la place à l'agir communicationnel et à ses appuis cognitifs («La révolte et les reproches qui s'expriment à l'encontre de la violation des normes ne peuvent s'appuyer que sur un contenu cognitif $\left.{ }^{11} »\right)$. Conçue comme «origine» d'un processus communicatif, l'humiliation échappe en tant qu'expérience avec sa propre processualité et ses contingences.

Ainsi, l'indignation exprimée «vis-à-vis de l'injustice révoltante qu'autrui a commise à mon endroit ${ }^{12}{ }^{2}$ et appelant les excuses de l'offenseur n'est, face à l'humiliation, qu'une réaction possible parmi d'autres. Et, quand on y songe, une réaction plutôt singulière. Premièrement, elle suppose qu'une humiliation puisse être «réparée» par des excuses, quand d'aucuns penseront qu'une conduite humiliante est précisément inexcusable ${ }^{13}$, à plus forte raison lorsqu'il

9. Peter Strawson, «Freedom and Resentment», Proceedings of the British Academy, vol. 48, 1962, p. 1-25.

10. Jürgen Habermas, Morale et communication, op. cit., p. 65-66.

11. Ibid., p. 69.

12. Ibid., p. 66 .

13. John L. Austin, Écrits philosophiques, Paris, Le Seuil, 1994 (1962), p. 158. 
est manifeste que l'humiliation a été causée intentionnellement. Deuxièmement, elle suppose que l'offensé ait toujours foi dans la force illocutoire de son discours, dans le fait que l'humiliation - l'humiliation publique en particulier - n'a pas anéanti la force illocutoire de ses sommations et vitupérations. Bien souvent, l'humiliation se poursuit en effet à travers les réactions désordonnées de l'offensé (fébrilité, rougissement, bégaiement, postillons, etc.) et ses tentatives - rapidement perçues comme hystériques - d'obtenir réparation. À la suite d'une humiliation, n'importe qui ne peut prendre la parole d'une manière qui ne continue d'être humiliante. Ainsi, il semble que le seul modèle de réaction à l'humiliation évoqué dans cet extrait par Habermas ne vale pas pour «quiconque, soit-il l'homme le plus endurci»; il vaudrait surtout pour «l'homme le plus endurci» ${ }^{14}$, l'homme ayant la force - la faiblesse? - de dépasser, sur le plan pratique et expressif, la honte qu'il ressent, ou de conjurer la honte qu'il devrait ressentir face à l'humiliation.

Tout autre individu doit compter avec la honte. Si l'humiliation causée par autrui peut être conçue sur le plan affectif comme un sentiment de honte injustement infligé, c'est-à-dire comme le mélange d'un sentiment d'injustice et d'un sentiment de honte, les implications praxéologiques du premier (indignation, reproches, quête de justice, etc.) peuvent difficilement être poursuivies en ignorant les implications pathiques du second. Quelles sont-elles? Dans sa définition de la honte, le sociologue phénoménologue Jack Katz identifie pas moins de dix composantes:

Shame is (1) an eerie revelation to self that (2) isolates one (3) in the face of a sacred community. What is revealed is a (4) moral inferiority that makes one (5) vulnerable to (6) irresistible forces. As a state of feeling, shame is (7) fearful, (8) chaotic, (9) holistic and (10) humbling ${ }^{15}$.

Dans un texte précédent consacré au «meurtre de bon droit» (righteous slaughter), Katz avait montré comment l'humiliation pouvait être transcendée et comme renversée dans l'accès de rage; une attitude émotionnelle par laquelle celui qui a été rabaissé (driven down) se relève soudainement (rise $u p$ ); une attitude d'autant plus immédiatement accessible que la rage partage bien des qualités (caractère chaotique, holistique, irrésistible) avec la honte qui a envahi l'individu: "by becoming humiliated, a person has already constructed certain fundamental elements of rage ${ }^{16} »$.

L'humiliation, dans la reconnaissance phénoménologique de sa dimension de honte, dispose à la rage et à la violence, certainement autant ou davantage qu'à la discussion ou la dispute éthique. C'est que l'enragé perçoit

14. J. Habermas, Morale et communication, op. cit., p. 66.

15. Jack Katz, How Emotions Work, Chicago, University of Chicago Press, 1999, p. 147.

16. Jack Katz, Seductions of Crime. Moral and Sensual Attractions in Doing Evil, New York, Basic Books, 1988, p. 26. 
peut-être mieux que le sujet imaginé par Habermas que l'humiliation et la honte l'ont - comme y insiste Katz - «isolé de la communauté de référence», rendant ses actes de langage très incertains dans leurs capacités illocutoire et perlocutoire. Le lien illocutoire que l'on cherche à établir avec des tiers par l'indignation, le blâme, etc., suppose une reconnaissance pré-discursive, précisément ce que l'humiliation a mis à mal.

La violence n'est elle-même qu'une réaction contingente à la suite d'une humiliation. Dans bien des cas, la réaction affective se limitera indéfiniment au versant pathique de la honte et de la peur paralysante qui l'accompagne. La crainte d'avoir disparu socialement n'engage pas nécessairement à «agir», qu'il s'agisse ici d'engager une dispute éthique ou d'en venir aux mains. L'humiliation, comme d'autres "événements difficiles à encaisser» ${ }^{17}$, a chez nombre d'entre nous des effets déconcertants, incapacitants ${ }^{18}$.

Ainsi, un basketballeur ayant été victime d'un facial dunk (un «smash» reçu en pleine tête après une tentative ratée de contrer l'adversaire) pourra perdre ses moyens et être coupé dans ses effets pour un bon moment. La victime de ce tour de force, affectée par la supériorité éclatante de son adversaire direct, peut certes réagir violemment, par une faute flagrante indiquant sa frustration. Mais le geste humiliant peut aussi provoquer ce qu'il vise littéralement: intimider l'adversaire, inhiber son action, le forcer à l'humilité. L'humilié pourra dans ce cas «se faire tout petit», "se montrer inexistant» pour le reste de la partie. L'humiliation a alors également des conséquences pour les partenaires de l'humilié, des conséquences sociales. Au-delà de la subjectivité blessée du sportif qui s'en ressent victime, c'est, à travers l'(in)action de celle-ci, la partie elle-même qui est compromise. Imaginons une autre scène, dans les circonstances d'un repas entre amis, monsieur, après s'être senti humilié par un commentaire maladroit de madame, devient «fantomatique» pour le reste de la soirée. Cela ne manque pas d'être remarqué, de "pourrir l'atmosphère», de "plomber l'ambiance» et éventuellement de provoquer le départ anticipé des convives. Rien ne remplace ici les descriptions phénoménologiques de Heidegger, dont le concept de Stimmung, signifiant à la fois l'humeur et l'ambiance, a cet intérêt de saisir pleinement, dans sa dimension individuelle et dans sa dimension sociale, l'expérience faite ensemble d'une passion triste comme l'humiliation:

17. Joan Stavo-Debauge, «Des événements difficiles à encaisser. Un pragmatisme pessimiste», dans D. Cefaï, C. Terzi (dir.), L'expérience des problèmes publics. Perspectives pragmatistes, Paris, EHESS (Raisons pratiques, 22), 2012.

18. On peut ici citer cet exemple donné par Freud: «Un jour, [...] mon père me raconta le fait suivant: "une fois quand j'étais jeune, dans le pays où tu es né, je suis sorti dans la rue un samedi, bien habillé et avec un bonnet de fourrure tout neuf. Un chrétien survint; et d'un coup, il envoya mon bonnet dans la boue en criant: Juif, descends du trottoir!" - Et qu'est-ce que tu as fait?- "J'ai ramassé mon bonnet", dit mon père avec résignation» (Sigmund Freud, L'interprétation du rêve, Paris, PUF, 2012 [1900], p. 203). 
Une tristesse envahit l'être humain avec lequel nous nous trouvons. [...] L'être devenu triste se ferme, il devient inaccessible [...]. Tout est comme d'habitude et est pourtant autre, et pas seulement à tel ou tel point de vue : [...] c'est plutôt le "comment" dans lequel nous sommes ensemble qui est autre. [...] L'homme attristé nous fait entrer dans le mode sur lequel il est, sans que nous-mêmes devions nécessairement être tristes. L'être en commun [...] est autre, il a changé de ton. [...] Il apparaît ceci : la tonalité est si peu à l'intérieur d'une quelconque âme de l'autre, si peu aussi $[\ldots]$ dans la nôtre, qu'il vaut mieux dire $[\ldots]$ : cette tonalité s'étend à présent sur tout. [...] La tonalité n'est pas un étant qui se rencontre dans l'âme en tant qu'impression vécue, mais elle est le comment de notre Dasein commun ${ }^{19}$.

Le ressentiment n'est pas juste un indicateur de l'existence d'une «dimension morale» dans l'humiliation et, à ce titre, le possible déclencheur d'une discussion éthique, comme on pourrait le retenir des quelques pages qu'y accorde Habermas dans Morale et communication. Le ressentiment n'est jamais un simple motif pour la communication. Il s'active toujours dans la communication et la travaille de l'intérieur. On assiste avec lui à «l'élaboration d'une expérience négative ${ }^{20}$ », dans laquelle ce dont précisément il est fait expérience, c'est une incapacité à l'action communicative, une indisposition qui peut amener la personne à érupter dans la rage ou à se murer dans le silence. On trouvera alors excessive cette prétention de Habermas d'avoir mis au point une perspective sur l'activité communicationnelle par laquelle il est enfin possible de «percevoir les phénomènes moraux dans leur plénitude ${ }^{21}$. Ce qui apparaît clairement aux commentateurs sportifs et aux convives d'un dîner raté devrait être accessible à Habermas: en tant que modèle de rationalité et, ultimement, de démocratie, la théorie de l'agir communicationnel appelle une théorie du pâtir communicationnel.

\section{Sensibiliser Habermas?}

L'attention qu'accorde Habermas dans TAC aux affects et à la dimension sensible de la communication peut être considérée comme insuffisante, c'està-dire améliorable. Nous ne nions aucunement l'intérêt majeur de l'œuvre et ne prétendons pas lui opposer, à travers une critique externe, une théorie alternative jugée supérieure. À nos yeux, le théoricien allemand a élaboré ces quarante dernières années l'ensemble théorique le plus complet et le plus convaincant, en théorie sociale et en philosophie politique. Beaucoup sont ceux qui le critiquent; rares sont ceux qui peuvent prétendre le concurrencer.

19. Martin Heidegger, Être et temps, Paris, Gallimard, 1992 (1927), p. 106-107.

20. Erving Goffman, Les cadres de l'expérience, Paris, Minuit, 1991.

21. Jürgen Habermas, Morale et communication, op. cit., p. 64. 
Dans ce texte, nous tentons de poser quelques jalons pour une «critique constructive» de la TAC sur le plan de son rapport au sensible, c'està-dire une critique qui cherche à construire à partir d'une théorie existante, plutôt qu'à la démanteler. Cette tentative est alors également celle d'une «critique interne»; elle cherche à retrouver à l'intérieur même de la théorie en question des références et ressources existantes qui, si elles étaient davantage déployées ou mieux agencées, permettraient à la théorie d'ensemble d'étendre sa portée. La critique que nous tentons consistera à sensibiliser la TAC, à rechercher les suites de l'éthique de la discussion dans une esthétique de la communication, à prolonger la théorie de l'agir communicationnel par une théorie du pâtir communicationnel ${ }^{22}$. «Sensibiliser Habermas» signifiera alors trois choses:

Premièrement, en réexaminant la théorie du langage prise en référence par Habermas dans TAC, le «modèle instrumental du langage» du psycholinguiste autrichien Karl Bühler, nous chercherons à en recouvrer l'intelligence sémiotique; le modèle de Bühler insistant en effet beaucoup plus que ne le fit Habermas sur l'opération «simultanée et équiprimordiale» des fonctions expressives, cognitives et appellatives du langage, sur leurs interactions ou interférences, et donc sur le caractère souvent instable, trouble et indécidable des processus de communication ${ }^{23}$. La communication langagière est pour Bühler une affaire sensible, un «parcours complexe» et moins transparent que le pose Habermas.

Ensuite, par un recours au pragmatisme sémiotique de Charles Sanders Peirce - autre inspiration majeure dans l'œuvre de Habermas depuis Connaissance et intérêt ${ }^{24}$ - il s'agira de préciser les voies d'une sortie du «logocentrisme» pour la TAC. Progressivement rabattu sur le Diskurs et l'argumentation, l'agir communicationnel selon Habermas apparaît réduit à un «agir linguistique ${ }^{25}$ ». L'agir communicationnel gagnerait en effet à se concevoir et à s'observer à de moindres niveaux d'élaboration discursive, à travers les usages les plus ordinaires du langage, voire à partir de processus sémiotiques dans lesquels le langage est marginal ou absent. En nous référant aux catégories phénoménologiques de Peirce (priméité, secondéité, tiercéité) et en les appliquant aux interactions communicatives, nous montrerons que la sémiose peut opérer selon trois modes ou canaux distincts.

22. Nous avons été inspiré dans cette entreprise par des textes tels que Les grammaires de l'intelligence de JeanMarc Ferry (Paris, Cerf, 2007), L'esthétique de la communication d'Herman Parret (Paris, Vrin, 1999), ainsi que par Jean-Louis Genard, qui nous a introduit à Peirce.

23. Karl Bühler, Théorie du langage, Paris, Agone, 2009 (1934).

24. Jürgen Habermas, Connaissance et intérêt, Paris, Gallimard, 1973 (1968).

25. Lenore Langsdorf «Reconstructing the Fourth Dimension: A Deweyan Critique of Habermas' Conception of Communicative Action», dans M. Aboulafia, M. Bookman, C. Kemp (dir.), Habermas and Pragmatism, Londres/New York, Routledge, 2002. 
Derrière les signes abstraits et les interprétations logiques (tiercéité) auxquels se limite la TAC, nous rappellerons l'importance des signes concrets et des «interprétants énergétiques» (secondéité), mais aussi des signes vagues, des sensations, sentiments et autres «interprétants émotionnels» (priméité).

Enfin, notre critique sollicitera un troisième auteur important pour Habermas, lui aussi pragmatiste, George Herbert Mead, pour rappeler avec lui l'importance, pour la communication, des corps qui la supportent et des milieux qui l'accueillent et la reçoivent. Si l'œuvre de Mead est déterminante pour Habermas, qui lui emprunte sa conception d'une «communication idéale» fondée dans une «intersubjectivité intacte ${ }^{26}$ », Habermas manque de considérer que chez Mead, les rapports intersubjectifs sont plongés dans des «mondes sociaux», des «univers de discours» et des «champs d'expérience» ${ }^{27}$. L'intention de «mise en situation de la raison» affichée par Habermas ne va pas suffisamment loin; on trouve chez Mead les moyens conceptuels de dépasser l' «universalisme mince» du théoricien allemand, à travers ce que Mitchell Aboulafia propose d'appeler un «contextualisme critique ${ }^{28} »$.

\section{Une théorie attentive à l'instabilité de la communication}

\section{Fonctions du langage et prétentions à la validité}

En prétendant réaliser dans $T A C$ une «mise en situation de la raison», Habermas entendait rappeler qu'une théorie de la signification ne peut se limiter à une «sémantique formelle» (Frege), et nier ses dimensions pragmatique et intersubjective. Pour développer sa «critique de la théorie de la signification» ${ }^{29}$, le théoricien allemand recourt au «modèle instrumental du langage» (Organon-modell) de Karl Bühler. Le psycholinguiste autrichien conçoit le langage avant tout comme un «instrument» (organon) permettant d'interagir avec les autres ou d'agir sur les autres par la médiation de signes; un moyen pour un «émetteur» d'entrer en relation avec un «récepteur» par le biais des signes et leur capacité à représenter des objets et états de choses. En s'appuyant sur Bühler, Habermas élabore sa théorie de la signification à partir d'une conception du langage qui l'inscrit directement dans la situation d'interlocution, une conception pragmatique et intersubjective qui s’intéresse avant tout à la

26. J. Habermas, TAC 2, p. 8

27. Daniel Cefaï, «Mondes sociaux», SociologieS, 2015.

28. Mitchell Aboulafia, «Habermas and Mead: On Universality and Individuality», Constellation, vol. 2, no 1, 1995, p. 94-113.

29. J. Habermas, TAC 1, p. 283-289; J. Habermas, «Toward a Critique of The Theory of Meaning», dans Postmetaphysical Thinking, Cambridge, MIT Press, 1992. 
relation établie dans le langage entre les interlocuteurs ${ }^{30}$, à ce que Habermas appelle le «lien illocutoire».

Cette relation établie entre un locuteur et son interlocuteur a plusieurs dimensions, et le langage, plusieurs fonctions. Pour Bühler, communiquer c'est en effet faire trois choses: «représenter» des objets et états de chose du monde, certes, mais également «exprimer» son état, ses intentions, et «appeler» chez l'autre une certaine attitude ou réaction. Cette tripartition des fonctions langagières de Bühler (représentation, expression, appel), fonde et organise la théorie habermassienne des «prétentions à la validité».

Pour Habermas, s'engager dans une interaction communicative (au sens propre de $T A C$, c'est-à-dire en suivant une «raison communicationnelle» plutôt qu' «instrumentale» ou «stratégique»), c'est entretenir certaines «prétentions à la validité» de ses propres énonciations et entretenir des attentes quant au fait que les interlocuteurs s'essaient eux-mêmes à des contributions valides. Ces prétentions universelles à la validité sont la vérité, la véracité, la justesse :

Lorsqu'un locuteur fait un énoncé, lorsqu'il affirme, raconte, explique, présente, prédit, discute quelque chose, il cherche avec l'auditeur un accord fondé sur la reconnaissance d'une prétention à la vérité. Lorsque le locuteur exprime dans une phrase une expérience vécue, lorsqu'il dévoile, délivre, confesse, révèle quelque chose, un accord ne peut intervenir que sur le fondement de la reconnaissance d'une prétention à la véracité. Lorsque le locuteur donne un ordre ou fait une promesse, promeut ou admoneste, un accord dépendra de la justesse que les participants auront à faire valoir pour cette action ${ }^{31}$.

On le remarque, ces trois prétentions à la validité correspondent aux trois fonctions identifiées par Bühler dans son «modèle instrumental du langage»: les fonctions cognitive (vérité), expressive (véracité) et appellative (justesse). Présentons-les, ainsi que les différents aspects du signe langagier qu'elles révèlent.

\section{Symbole, symptôme, signal: trois aspects du signe dans la communication}

La fonction cognitive - fonction de désignation ou de représentation (Darstellung) - du langage est celle par laquelle un locuteur réfère aux objets et états de choses dans le monde. C'est la fonction du langage "reliée au pôle de l'objectivité dans la situation de communication ${ }^{32}$ ». Pour sa fonction de représentation, le signe est considéré comme «symbole» (Bühler donne ici à «symbole» ce sens général de signe représentant son objet, qui s’éloigne

30. Pauline Marthelot, Karl Bühler. Du contexte à la situation, la signification, Paris, Armand Collin, 2012.

31. J. Habermas, TAC 1, p. 317.

32. P. Marthelot, Karl Bühler, op. cit., p. 11. 


\section{Figure 1}

Fonctions du langage (Bühler) et prétentions à la validité correspondantes (Habermas)

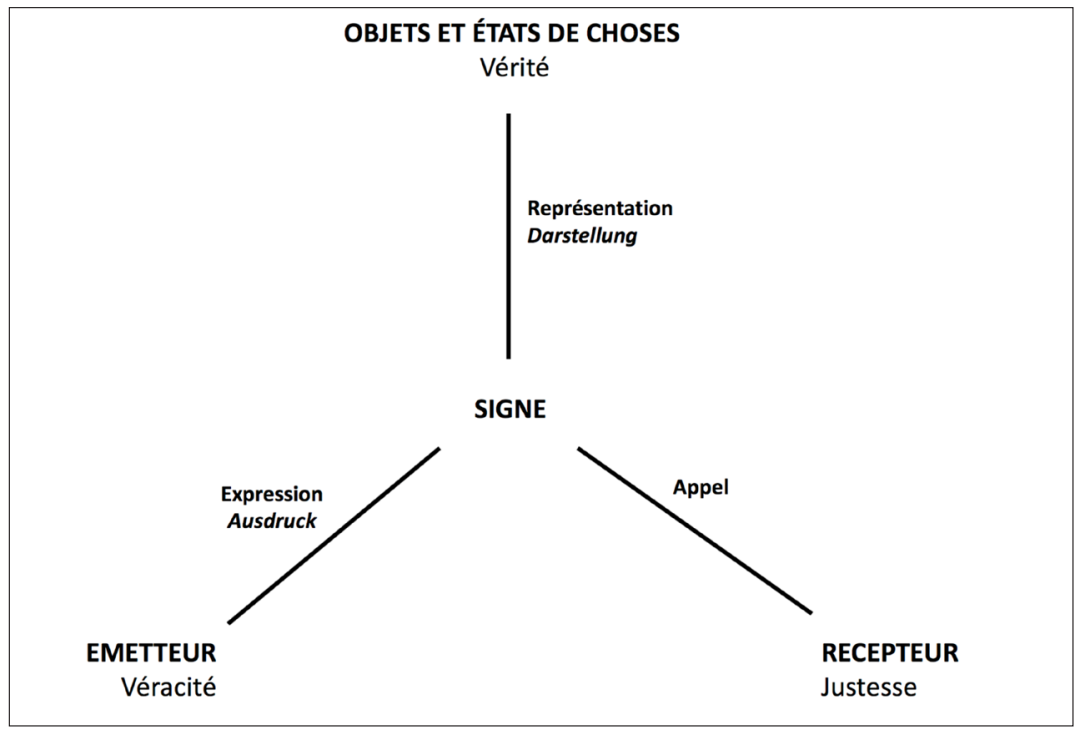

du sens plus spécifique que lui donne Peirce, nous le verrons). La fonction de manifestation (Kundgabe) ou d'expression (Ausdruck) est la fonction par laquelle un locuteur manifeste un état intérieur, exprime un vécu psychique. Dans sa fonction de manifestation des états du locuteur, tout signe constitue un «symptôme». Enfin, la fonction de déclenchement (Auslosung) ou d'appel (Appell) est celle par laquelle le locuteur cherche à influencer les vécus psychiques du récepteur, à éveiller son attention et à provoquer en lui un comportement adapté33. Bühler dit ici qu'on peut parler de speech appeal, comme on parle de sex appeaß ${ }^{4}$. Il faut que ce qu'émet le locuteur stimule le récepteur, que ça «lui parle», que ça «lui dise». Pensé sous la fonction appellative, le signe est «signal».

De la même manière que chez Peirce concernant l'icône, l'indice et le symbole, il ne faut pas, chez Bühler, considérer le symptôme, le signal et le symbole comme trois classes de signes, mais plutôt comme différents aspects

33. Notons ici que cette fonction d'appel ne se limite pas aux modalités de la séduction, de l'influence ou de la menace, dans une conception stratégique de la communication. Elle importe également dans la perspective d'un agir orienté vers l'intercompréhension, où «on en appelle» à la raison de l'interlocuteur, on cherche à provoquer chez lui la compréhension et l'assentiment (cf. 4.2., infra).

34. K. Bühler, Théorie du langage, op. cit., p. 110. 
du signe. Dans sa théorie des trois aspects (Dreiaspektenlehre), «Bühler met en évidence le fait que les trois fonctions du langage se réalisent à partir d'un même phénomène, considéré depuis des points de vue distincts ${ }^{35}$ ». "Par conséquent, un même signe peut être compris tantôt comme symptôme d'un état intérieur (dans la réalisation de la fonction de manifestation), tantôt comme signal guidant le comportement doué de sens (dans la fonction d'appel), tantôt comme symbole dans la fonction de représentation ${ }^{36}$.» Les fonctions du langage ne sont pas seulement multiples; dans le modèle sémiotique de Bühler, elles sont aussi simultanées: «les phénomènes langagiers sont de part en part sémiotiques ${ }^{37}{ }$.

\section{Les fonctions du langage, entre simultanéité et pertinence}

Si Habermas a bien retenu du modèle de Bühler la question de la triplicité des fonctions du langage, il n'a peut-être pas suffisamment honoré celle de leur simultanéité. Même s’il écrit que «les actions langagières sont toujours insérées dans un réseau complexe de rapports au monde ${ }^{38}$ » et "peuvent toujours être récusées sous chacun des trois aspects ${ }^{39}$ », Habermas fonde sa pragmatique universelle à partir de «cas idéalisés ou cas purs d'actes de parole $e^{40}$ ». Il s'intéresse surtout à montrer comment, aux trois fonctions identifiées par Bühler, correspondent différentes «classes» d'actes de langage, reprises à Austin et à Searle. Il distingue ainsi des «actions langagières expressives», «constatives» et «régulatrices», pour lesquelles la fonction de manifestation, ou de représentation, ou d'appel est prépondérante, et qui engagent donc aussi chaque fois une prétention à la validité spécifique: prétention de véracité, ou de vérité, ou de justesse normative.

Classifier de la sorte les actes de langage en assignant à chacun une fonction du langage et une prétention à la validité constitue sans doute un exemple du «fourvoiement substantialiste» que cherchait à éviter Bühler. À l'examen de nombreux actes de langage ordinaires, «les différentes prétentions à la validité [formulées par Habermas] ne peuvent être suffisamment discriminées les unes des autres ${ }^{41}$ ». Le rattachement d'une énonciation à une classe d'actes de langage par la primauté donnée à une fonction sur une autre est le fait de l'observateur-analyste, qui par là même «dé-sémiotise» le modèle. Les énonciations en elles-mêmes ne produisent que des signes, qui peuvent être perçus

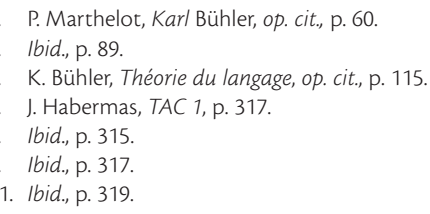


ou non, sélectionnés ou non, donner lieu à des interprétations très variables, des réinterprétations. Devant la simultanéité des fonctions, des distinctions ont bien lieu, mais elles sont le fait des partenaires de l'interlocution, dans la situation. C'est l'idée de «pertinence abstractive» qu'introduit Bühler: face à une énonciation calibrée d'une certaine manière, par un locuteur qui peut chercher à y faire primer une fonction sur une autre, le récepteur fait preuve d'une attention sélective et opère ses propres «prélèvements» :

Les systèmes psychologiques des partenaires de parole produisent et traitent le flatus voci d'une manière toute différente de celle que présuppose l'ancienne formule simpliste. En tant que récepteurs, les systèmes psycho-physiques sont des sélecteurs, et ils fonctionnent selon le principe de la pertinence abstractive [;] en tant qu'émetteurs, ces systèmes psychophysiques sont des stations de formatage. Tous les deux font partie de l'agencement propre à la communication par signaux ${ }^{42}$.

$\mathrm{Si}$, à travers le «formatage» donné à sa prise de parole, l'émetteur peut chercher à placer l'accent sur une fonction particulière du langage, l'énonciation peut être reçue sous un autre aspect, le récepteur y «prélevant» au final ce qui compte pour lui. Je tente de profiler mon énonciation de manière à faire primer la fonction d'appel, de soigner mon speech appeal, d'envoyer des «signaux»; mon interlocuteur saisit l'énonciation sous sa fonction de manifestation et y voit un comportement «symptomatique». Je peux chercher, pardessus tout, à m'exprimer avec véracité et à manifester ma subjectivité dans une situation où, malheureusement, il sied (justesse) d'adopter une attitude objectivante et de s'en tenir aux faits, où les énonciations ne seront appréhendées qu'à l'aune de la fonction cognitive et de leur prétention à la vérité.

Il serait intéressant, à l'intérieur d'une théorie de l'agir communicationnel, de rétablir la radicalité sémiotique de l'Organon-modell. La considération par Habermas de «cas purs d'actes de parole» limite son attention aux opérations et préférences de l'émetteur: la classification qu'il opère «fait ressortir l'aspect de validité sous lequel le locuteur aura préférentiellement compris son expression ${ }^{43}$ ». Or les préférences du récepteur sont tout aussi déterminantes dans la menée, le succès ou l'échec d'actes de parole. Il manque à la théorie de l'agir communicationnel une véritable théorie de la réception, une «théorie du pâtir communicationnel» qui s'intéresserait aux dispositions des interlocuteurs à prêter attention, à entendre, écouter et retenir (ainsi qu'aux milieux et aux situations qui les y disposent plus ou moins, comme nous y insisterons plus loin avec Mead). La dimension pathique de la communication à l'œuvre dans la réception n'est pas tant affaire de passivité que

42. K. Bühler, Théorie du langage, op. cit., p. 108-109.

43. J. Habermas, TAC 1, p. 317. 
de passibilité (et parfois d'impassibilité) ${ }^{44}$ : parmi le flot de ce qui est émis, il nous faut nous inquiéter de ce qui passe. Déplacer le centre de gravité de la théorie vers le pôle de la réception semble en effet la condition d'une réelle pragmatique de la communication: "La théorie des actes de discours a donné des privilèges certains à la génération et à la production du sens, sur la question de la compréhension et de l'interprétation». Il nous faudrait «renverser ce privilège» et commencer par «la transposition sémantique que tout acte d'interprétation réalise» ${ }^{45}$.

\section{Le fond trouble de l'interlocution}

La théorie de Bühler nous rappelle donc que la sémiose réalisée par la communication langagière est un "parcours complexe, où l'appel adressé à un autre donne le coup d'envoi d'un procès d'inter-compréhension qui devra traverser différentes dimensions du sens, effectuer le cercle complet au terme duquel les partenaires sont mis en situation de thématiser ensemble [...] la portée significative de leur communication ${ }^{46}{ }$. La communication est donc loin d'avoir chez Bühler la transparence qu'elle a chez Habermas.

Il faut se souvenir ici que Bühler est avant tout psychologue. S'il vient à la linguistique, ce n'est que pour articuler plus systématiquement les objets d'une psychologie qui conçoit «l'action comme un pilotage du sujet dans un monde de déterminations complexes et contradictoires ${ }^{47}$ ». À travers la mise au jour de la triplicité des fonctions du langage, il vise surtout l'intégration psychologique de trois «groupes d'interventions» qui interviennent dans la menée de l'action, qui la traversent en même temps qu'ils la contrarient ou la «dévient»:

Dans le cadre de sa relation au monde, le sujet doit réagir à trois groupes d'interventions. Le premier groupe est composé par ses propres perceptions qui fonctionnent en tant que signes de ce que l'on pourrait dire concernant les choses du monde. Le deuxième groupe est formé par des valeurs comme la connaissance de la vérité, le désir de bonheur, etc., qui sont intériorisées au cours de la vie par chaque sujet. Le troisième intervenant c'est l'autre, c'est l'influence réciproque et sensée du comportement des membres d'une communauté. Ces trois intervenants font que le but et sa réalisation ne cö̈ncident jamais; ils se défont an contraire continuellement. [...] Il existe donc une «synapse» comme dit Bühler,

44. "Passible signifie "capable de pâtir, de subir"; et cette capacité implique une activité, immanente à l'épreuve qui consiste à ouvrir son propre champ de réceptivité» (Henri Maldiney, «De la transpassibilité», Penser l'homme et la folie, Grenoble, Millon, 2007, p. 265).

45. Herman Parret, L'esthétique de la communication. L'au-delà de la pragmatique, Paris, Vrin, 1999, p. 8.

46. J. M. Ferry, Les grammaires de l'intelligence, op. cit., p. 98.

47. Janette Friedrich, "Quelques réflexions sur le caractère énigmatique de l'action», dans J.-M. Baudouin, J. Friedrich (dir.), Théories des l'action et éducation (Raisons éducatives, n 4, 2001, Bruxelles, DeBoeck, p. 106. 
entre le but et sa réalisation, une faille qui les sépare et les relie en même temps et qui, par conséquent, contraint le sujet à piloter ${ }^{48}$.

Ce qui est dit au sujet de l'action vaut pour la communication. Les tensions entre ces trois «bases relationnelles» (vis-à-vis du monde externe, vis-à-vis de soi et vis-à-vis de l'autre), qui sous-tendent les trois fonctions de langage, font et défont la communication. Nous l'avons dit, les trois fonctions du langage ne peuvent être assignées exclusivement à telle ou telle classes d'actes. Mais, une fois reconnue leur simultanéité dans le langage, on ne peut pas non plus penser celle-ci comme simple complémentarité, selon laquelle ces trois bases relationnelles coexisteraient tranquillement en tant que dimensions différentes de la communication. Elles sont prises au contraire dans des rapports de tension, de concurrence et d'interférence, qui donnent lieu à des sémioses d'autant plus troubles et instables qu'elles dépendent des sélections opérées, toujours avec un certain degré d'opacité mutuelle, par le locuteur et le récepteur. Plutôt que d'offrir trois «piliers» à la communication, les bases relationnelles du rapport aux objets, du rapport à soi et du rapport à l'autre agissent comme des forces polarisantes et déstabilisatrices: elles ne «fondent» la communication qu'en tant que processus fondamentalement instable.

On comprend alors mieux les implications des métaphores proposées par Bühler, du langage comme «instrument de guidage», et du sujet de l'action comme «pilote» ${ }^{49}$. Ces termes sont ici à interpréter dans leur sens fort; faute de guidage, de pilotage, les tendances internes à l'action la font se perdre ou chavirer:

Beaucoup de déraillements seraient explicables par le fait que le réalisateur est attiré dans le cercle de fascination d'un système étranger à son but, comme une comète est attirée d'un système solaire à un autre. [...]. Dans l'être humain, il existe tellement de systèmes étrangers et souvent contradictoires les uns par rapport aux autres, que le problème central d'une analyse de la structure est de savoir comment à travers ces systèmes un bateau peut être pilote vers des buts lointains et éloignés ${ }^{50}$.

Résumons. Nous venons de voir avec Bühler que le caractère simultané et équiprimordial des trois fonctions du langage (représentationnelle, expressive

48. Janette Friedrich, «Crise et unité de la psychologie. Un débat dans la psychologie allemande des années 1920», Bulletin de psychologie, $n^{\circ}$ 52, 1999, p. 254-255.

49. Cette psychologie de l'action et de la communication inquiète des «contradictions» et «déraillements» qui leur sont inhérents semble finalement assez éloignée de la conception plus confiante que se fait Habermas de l'interaction communicative. Ce dernier prend-il la mesure de la signification «instrumentale» - un épithète qui n'a pas vraiment les faveurs de Habermas quand il est question de communication ! - que Bühler donnait au langage? Pour Habermas, le langage est en effet loin de se limiter à un outil, un instrument dont je me saisis pour guider l'autre et me guider moi-même. Il est, bien plus, un médium - c'est-à-dire aussi un «milieu» au sein duquel j'évolue - constitutif d'un monde en commun (le «monde de la vie») qui nous saisit, mon interlocuteur et moi.

50. Karl Bühler, cité dans J. Friedrich, «Quelques réflexions sur le caractère énigmatique de l’action», op. cit., p. 106. 
et appellative) permettait de considérer un même signe sous son aspect de symbole, de symptôme ou de signal. Le fait qu'une énonciation puisse être simultanément symbole, symptôme et signal, que l'aspect du signe primant dépende du phénomène de "pertinence abstractive» et de l'écart entre les sélections du locuteur et les sélections des interlocuteurs, fait de la communication interpersonnelle un processus instable, toujours susceptible de «dérailler», et ce, sans que les parties mêlées en soient nécessairement conscientes. "Sensibiliser Habermas», à partir de cette première relecture de Bühler, revenait à insister sur le fait que la communication intersubjective ne va pas de soi. Elle est en elle-même une affaire sensible, délicate, touchy, dont les productions peuvent rester énigmatiques. Politiquement, cette conception radicalement sémiotique, insistant sur l'instabilité du processus communicationnel, invite sans doute à davantage de prudence et de réserve quant à la viabilité d'un espace public de discussion.

\section{Une théorie attentive à la pluralité des modes sémiotiques}

Après avoir placé l'accent sur le caractère simultané et équiprimordial des fonctions et les façons dont cette lecture de l'Organon-modell vient inquiéter la vision habermassienne de la communication, nous laissons derrière nous la question des interférences entre fonctions pour nous intéresser aux subtilités sémiotiques du fonctionnement propre à chacune. Car, après tout, comment fonctionnent ces fonctions? Comment opère le signe appréhendé soit comme symbole, soit comme symptôme, soit comme signal? Ici, la théorie du signe de Peirce ${ }^{51}$, d'une richesse inégalée, permet de prolonger la Sprachteorie de Bühler, dont les analyses ont malgré tout privilégié la fonction de représentation, et qui en matière de communication s'en est tenue à la communication langagière. La lecture de Bühler ne permet pas en effet de dépasser la «théorie de l'agir linguistique» ${ }^{52}$ reprochée à Habermas, et ne nous amène pas suffisamment loin dans l'analyse des modes opératoires du signe-symptôme et du signe-signal.

Les opérations sémiotiques des trois fonctions de Bühler peuvent être spécifiées en appliquant, à chacune, les trois catégories phénoménologiques au fondement de la théorie du signe de C. S. Peirce ${ }^{53}$ : priméité, secondéité, tiercéité. À travers celles-ci, Peirce visait à distinguer trois manières de recevoir le monde: la «possibilité qualitative» éprouvée sur le mode du sentiment (priméité), le «fait actuel» reçu par la perception (secondéité), la «loi»,

51. Bühler ne semblait pas avoir connaissance des travaux de Peirce (W. Abraham, «Préface», dans K. Bühler, Theory of Language. The Representational Function of Language, Amsterdam, John Benjamins, 2011, p. xxxviii).

52. L. Langsdorf «Reconstructing the Fourth Dimension...», op. cit.

53. Charles S. Peirce, Écrits sur le signe, Paris, Seuil, 1978, p. 69 et s. 
la règle générale, dont nous faisons l'expérience à travers l'habitude et que nous appréhendons par la pensée et le langage (tiercéité).

La «théorie de l'agir communicationnel» tend à ne considérer le rapport à soi, au monde et à l'interlocuteur que médiatisé par les symboles, les signes généraux, c'est-à-dire sur le mode de la tiercéité. Ce faisant, elle néglige ce qu'il y a de plus «immédiat» dans la communication ${ }^{54}$, ses dimensions sensibles: le «sensible» du sensoriel (secondéité) et le «sensible» du sentiment (priméité). Ce sont ces dimensions que les catégories peirciennes nous permettent de recouvrer. Suivant une suggestion de Jean-Marc Ferry ${ }^{55}$, nous tentons d'intégrer les dimensions de priméité et secondéité au modèle de Karl Bühler, afin de le «sensibiliser» et, à travers lui, stimuler une conception enrichie de l'agir communicationnel. Présentons brièvement ces trois «catégories».

La priméité - sans doute la catégorie la plus difficile à saisir dans la mesure où précisément elle concerne l'insaisissable - est le mode d'être d'une qualité possible mais inactuelle. La noirceur peut être sur un mode premier. Elle n'«existe» pas, sur le mode du fait actuel, au sens où elle n'est pas cet objet noir-ci, ni cet animal noir-là, sur lequel notre attention visuelle se porte. Elle n'est pas non plus sur le mode général de la loi, de la règle, de la convention ou de l'habitude, qui veut que «le noir» est la couleur la plus sombre, se prononce \nwab\en français, s'écrit b-l-a-c-k en anglais, est la couleur du deuil, etc. La noirceur en soi, avant d'être incarnée dans des objets noirs (secondéité) et d'être socialement reconnue comme «le noir» (tiercéité), est une "possibilité qualitative sui generis» ${ }^{56}$.

Autre façon de suggérer la priméité, pensons à l'expérience que nous pouvons faire de la morosité d'une petite ville industrielle que nous traversons. Ce n'est pas cet immeuble délabré, ce trottoir sale, ce ciel pluvieux ou la faible fréquentation de cette rue, qui est morose. La morosité ressentie n'émerge pas d'informations et de connaissances au sujet de la ville en question, son nom, sa localisation, le fait que le taux de chômage y a dépassé en 2016 les $30 \%$ et que $20 \%$ des commerces ont fermé ces 10 dernières années, etc. Bien entendu, de tels éléments indiciels (signes seconds) et symboliques (signes troisièmes) interviennent dans l'expérience, mais, en elle-même, l'expérience de la morosité est structurée par l'iconique (signes premiers): elle

54. Eugene Rochberg-Halton, Meaning and Modernity. Social Theory in The Pragmatic Attitude, Chicago, University of Chicago Press, 1986, p. 24-40.

55. «La systématique de K. Bühler: symptôme/signal/symbole prend une valeur de référence équivalente à celle de la systématique de C. S. Peirce icône/indice/symbole. Ces deux systématiques me paraissent non seulement compatibles entre elles, mais complémentaires. Chacune se laisse reprendre et développer à la lumière de l'autre» (J. M. Ferry, Les grammaires..., op. cit., p. 17).

56. C. S. Peirce, Écrits sur le signe, op. cit., p. 70. 
se présente à travers un «sentiment absolument simple, l'émotion du tout ensemble $e^{57}$. Elle tient à une qualité de l'ambiance qui nous enveloppe ${ }^{58}$, une tonalité à laquelle nous nous accordons - au sens d'attunement - et dont nous venons à partager l'humeur, la Stimmung, sans nécessairement y prêter attention. Cette continuité qualitative et le fait que le sentiment en question ne soit pas relevé ou thématisé par la pensée, signent un rapport au monde en priméité. La priméité, écrivait Umberto Eco, nous amène «au seuil inférieur de la sémiotique» : l'affect confusément ressenti ne peut être imputé à tel ou tel fait affectant; l'impression ne retrouve pas son «objet imprimeur ${ }^{59}$ ».

L'idée de secondéité est plus «facile à comprendre. Celle de premier est si fragile qu'on ne peut la toucher sans la détruire, mais celle de second est éminemment dure et tangible ${ }^{60}{ }$. La secondéité caractérise une réception du monde sur le mode de l'«actualité» (à entendre au sens, en anglais, d'actually: «en fait»). La sécondéité est ainsi la catégorie phénoménologique de la perception nette de «tout ce qui frappe les sens». «Forcer», «heurter», «frapper», sont quelques-uns des termes utilisés par Peirce pour dénoter la «brutalité des faits», le caractère «percussif» des sémioses marquées par la secondéité, et la dimension de «lutte» (struggle) de ce rapport au monde. Cela nous fait remarquer que le sentiment ou la sensation évoquée pour la priméité ne peut avoir ce caractère «frappant». Le sentiment premier ne peut être que vague : nettement perçu il suppose la conscience de l'objet, de l'existant - soit, dans le langage de Peirce - du «second» qui le cause ; articulé, par la pensée et le langage, il ressort à la tiercéité.

La tiercéité désigne en effet la catégorie phénoménologique du général, du régulier, de l'habituel, du typique, qui vient faire médiation dans notre rapport au monde ${ }^{61}$ : «Nous avons vu que c'est la conscience immédiate qui est prééminemment première, la chose morte externe qui est prééminemment seconde. Pareillement, c'est la représentation médiatrice entre les deux qui est prééminemment troisième.»

Revenons à Bühler. Suivant le conseil de Peirce d' «appliquer (ces trois catégories) aux objets qu'il nous plaît», notre argument est que chacune des trois fonctions du langage qui structurent l'Organon-modell et qui fondent également la théorie de l'agir communicationnel de Habermas, peut elle-

57. Ibid., p. 88 («tout ensemble», en français dans le texte).

58. Comme le rappelle Jean-Paul Thibaud («Petite archéologie de la notion d'ambiance», Communications, 2012, 90), «le verbe latin ambire renvo[ie] au mouvement des deux bras lors d'une étreinte chaleureuse».

59. «L'iconisme primaire naturel serait la qualité propre d'empreintes qui n'ont pas encore trouvé (nécessairement) leur objet imprimeur, mais qui se tiennent prêtes à «reconnaître» cet objet» (Umberto Eco, Kant et l'ornithorynque, Paris, Grasset, 1999, p. 112).

60. C. S. Peirce, Écrits sur le signe, op. cit., p. 74.

61. Ibid., p. 76. 
Figure 2

Les « catégories» (Peirce) appliquées aux fonctions du langage (Bühler)

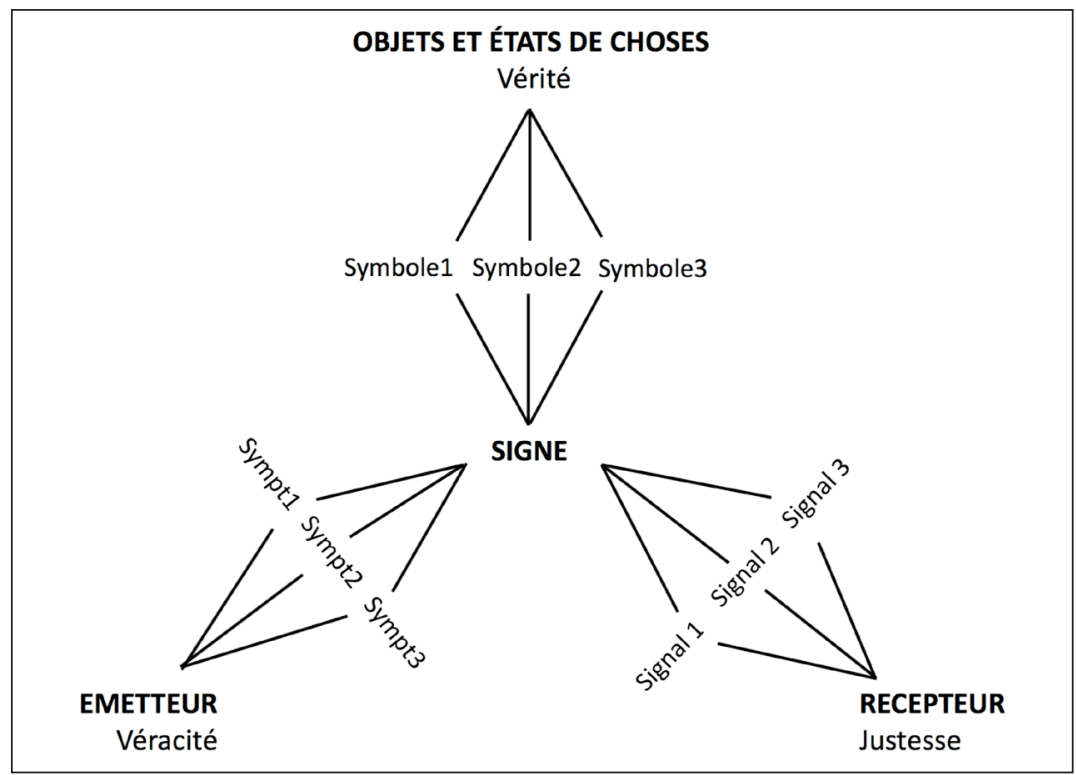

même opérer sur ces trois modes: possibilité, actualité, régularité. Pris pour sa valeur cognitive de (re)présentation, le signe peut renvoyer aux objets et états de chose sur ces trois modes. Saisi sous l'angle de l'expression, le signe peut symptomatiser l'état et le vécu psychique du locuteur sur ces trois modes. Considéré comme signal dans sa fonction d'orientation des états et comportements des interlocuteurs, le signe suscite des effets - ce que Peirce appelle «interprétant» - sur ces trois modes (voir la figure 2).

\section{Trois modes cognitifs}

La fonction cognitive du langage se déclinerait ainsi en trois fonctionnements. Ce que Bühler appelait lui-même «symbole», le signe saisi dans sa fonction désignative, se trouve triplé chez Peirce: «symbole» (tiercéité du rapport signe-objet), «indice» (secondéité), «icône» (priméité). Là où les symboles opèrent indirectement, par médiation, les signes indiciels et iconiques nous mettent plus immédiatement et sensiblement en rapport avec les objets et les états de chose du monde: que ce soit en les pointant physiquement, en les figurant par l'image ou encore en nous les «plaçant sous les yeux» métaphoriquement. Si, pour Habermas, on s'en souvient, la fonction 
cognitive de représentation de Bühler est couplée à la prétention à la validité de «vérité», le dispositif que nous proposons invite à étendre l'évaluation faite des énoncés quant à leur vérité aux communications structurées par l'indiciel ou l'iconique ${ }^{62}$.

En effet, si l'on peut (pré)tendre à la vérité au sujet de quelque chose à travers l'usage de signes généraux, de formes symboliques, propositionnelles, argumentatives, on peut aussi le faire sur un mode indiciel, ostensif, en montrant des choses, en pointant le doigt vers un existant présent, ou en produisant les traces de ce qui a existé, de ce qui a été «là» (empreinte, photographie, relique...). Certes, les indices n'engagent pas la question de la vérité de la même manière que les propositions constatives, mais il est certain que lorsque A s'adresse à B en criant "Derrière toi !» ou lorsqu'un feu passe au vert, A prétend qu'il y a vraiment matière à se retourner et le sémaphore «prétend» que la circulation venant des autres côtés est réellement à l'arrêt.

On peut également (pré)tendre à la vérité sur un mode iconique, sur le mode de la ressemblance, de l'association ou de l'évocation, par la juste figuration sensible de quelque chose dans le monde, sinon ineffable. Une esquisse, une phrase musicale, un vers, une métaphore, peuvent tendre à la vérité sur le mode du possible - c'est notamment l'idée de "vérité métaphorique» chez Ricoeur ${ }^{63}$. Ils tendent à la vérité sans prétendre à l'exactitude de ce qui est riche en indices (secondéité), ni à la détermination de ce qui est argumenté (tiercéité).

On distinguera donc trois modes pour la fonction cognitive du signe: représentation par le symbole, présentation par l'indice, mise en présence par l'icône. L'intelligence peut en venir à se spécialiser sur l'un ou l'autre de ces modes ${ }^{64}$. Tel les Grecs, qui insistaient sur la nécessité de compléter l'intelligence théorique par la poésis comme faculté d'iconisation et la phronésis comme capacité d'indexicalisation ${ }^{65}$, Peirce valorisait l'apport cognitif propre des facultés premières, secondes et troisièmes, et plaidait pour leur collaboration dans le travail phénoménologique d'identification des «modes d'être»:

The faculties which we must endeavor to gather for this work are three. The first and foremost is that rare faculty, the faculty of seeing what stares one in the face, just as it presents itself [...]. This is the faculty of the artist who sees for example the apparent colors of nature as they appear. [...] That artist's observational power

62. Pour de premières tentatives, dans le cadre d'analyses empiriques: Mathieu Berger, «Répondre en citoyen ordinaire. Pour une analyse ethno-pragmatique des compétences profanes», Tracés, 2008, p. 15; M. Berger, «La participation sans le discours. Enquête sur un tournant sémiotique dans les pratiques de démocratie participative», EspacesTemps, 2014; M. Berger, «The Politics of Co-Presence. An Ecological Approach to Resistance in Top-Down Participation», European Journal of Political and Cultural Sociology, vol. 2, nº 1, 2015.

63. P. Ricoeur, La métaphore vive, Paris, Seuil, 1975.

64. J. M. Ferry, Les grammaires de l'intelligence, op. cit.

65. Pieter Duvenage, Habermas and Esthetics. The Limits of Communicative Reason, Polity, 2003, p. 97. 
is what is most wanted in the study of phenomenology. The second faculty [...] is a resolute discrimination which fastens itself like a bulldog upon the particular feature that we are studying, follows it wherever it may lurk, and detects it beneath all its disguises. The third faculty we shall need is the generalizing power of the mathematician who produces the abstract formula that comprehends the very essence of the feature under examination purified from all admixture of extraneous and irrelevant accompaniments ${ }^{66}$.

Aux facultés de «voir le monde comme il apparaît», d'opérer des «distinctions résolues», d' «abstraire l'essence de la chose examinée» par un «pouvoir de généralisation», correspondent différentes attitudes cognitives, qui peuvent indiquer différentes sensibilités d'enquêteurs (celle de l'artiste, celle du bulldog alerte et tenace qui s'accroche aux détails de l'expérience, celle du mathématicien). Les modes premiers et seconds nous rappellent que la sensibilité a une dimension cognitive : la disposition sentimentale nourrit l'évocation iconique, l'éveil sensitif favorise les prises indicielles. Qu'en est-il du mathématicien? Son intelligence n'évolue pas à l'écart de toute sensibilité. Peirce évoque ainsi dans différents textes une esthétique des mathématiques, et l'émotion particulière ressentie à la découverte d'une parfaite démonstration. Il n'y a pas à choisir entre affects et raison, ni à les opposer.

Mais comment faire coexister et surtout faire communiquer ces «sensibilités», notamment dans l'arène politique, afin qu'une communication sémiotiquement enrichie puisse rationnellement - mais selon une rationalité élargie - coordonner l'action? Ici, il faut prendre garde de ne pas trop psychologiser l'idée de sensibilité, de la réifier à travers une sorte de caractériologie des intervenants potentiels. Les moods qui nous intéressent renvoient autant aux «humeurs» des interlocuteurs qu'aux différentes tonalités de la communication et à l'interaction de ses «modes», au sens grammatical (grammatical moods).

Ainsi, pour Peirce, recadrée dans le contexte de la grammaire du verbe, l'intelligence symbolique s'accomplit à l'«indicatif» (qui porte mal son nom tant il est par excellence le mode grammatical du symbole et non de l'indice): un signe symbolique déclare, affirme, asserte que x signifie y. Les icônes et les indices, de leur côté, «n'assertent rien»:

Si une icône pouvait être représentée par une phrase, celle-ci devrait être au mode potentiel, c'est-à-dire dirait simplement: "Supposons qu'une figure ait trois côtés, etc.». Si, de la même façon, on interprétait un indice, le mode serait impératif, ou exclamatif: «Voyez !» ou «Regardez!» ${ }^{67}$.

66. Charles S. Peirce, «On Phenomenology», The Essential Peirce, vol. 2 (1893-1913). Selected Philosophical Writings (édité par le Peirce Edition Project), Bloomington, Indiana University Press, 1998 (1903), p. 147.

67. C. S. Peirce, cité dans: Christiane Chauviré, Peirce et la signification. Introduction à la logique du vague, Paris, PUF, 1995, p. 99. 
Sur le plan des apports cognitifs propres à la priméité, à la secondéité et à la tierciété, il est possible de prolonger la trichotomie «icône - indice symbole» et sa traduction grammaticale «subjonctif - impératif - indicatif» par la trichotomie peircienne des inférences logiques «abduction - induction - déduction» ${ }^{68}$, qui proposent autant d'entrées possibles dans l'activité de raisonnement: par la possibilité (l'abduction comme imagination hypothétique), par l'actualité (l'induction comme traçage et collection de cas) et par la règle générale (déduction).

Reste alors à mener les enquêtes empiriques d'une « ethno-pragmatique ${ }^{69}$ » ou «ethno-sémiotique ${ }^{70}$ » de la communication qui permettraient d'observer et d'évaluer les formes de coordination et d'interaction établies entre ces différentes sensibilités cognitives, non seulement à un niveau interlocutoire, entre les partenaires de l'interaction communicative, mais aussi à un niveau intralocutoire, comme contributions composites d'un même communicant. Les moyens que nous avons de connaître le monde et de tendre à la vérité à son sujet empruntent tour à tour aux indices, aux icônes et aux symboles. Les sensibilités grammaticales, les moods de l'impératif, du subjonctif, de l'indicatif ne peuvent être complètement dissociés et alloués aux «bulldogs», «artistes» et «mathématiciens». Ces modes organisent conjointement notre raisonnement, qui n'a cesse de commuter entre inférences inductives, abductives et déductives. C'est cette composition dynamique qu'il faut s'efforcer de saisir dans les communications bien menées, rationnelles dans ce sens élargi, dans lesquelles les «prétentions à la vérité» des communicants s'appuient sur un «polyglottisme sémiotique», leurs aptitudes à représenter, à présenter et à mettre en présence.

68. "Abduction [...] is of the order of sentiment [...]. Induction, of course, is of the order of observational experience [...]; this strategy of knowledge corresponds to Secondness [...]. Deduction is of the order of thought: one infers a result from the application of a general rule to a particular case; this corre sponds to Thirdness [...]» (Herman Parret, Semiotics and Pragmatics. An Evaluative Comparison of Conceptual Frameworks, Amsterdam/Philadelphie, Benjamins, 1983, p.27). Notons que Peirce a fluctué dans sa présentation des rapports entre inférences fondamentales et catégories. Si nous reprenons ici, avec Parret, la succession «Abduction (Priméité) - Induction (Secondéité) - Déduction (Tiercéité)», la plus intuitive et la plus répandue, un ordre dans lequel les inférences progressent en certitude et diminuent en fécondité (uberty), Wim Staat propose une autre série, vers laquelle Peirce s'est orienté dans un second temps: «Abduction (Priméité) - Déduction (Secondéité) - Induction (Tiercéité)». L'abduction est ici pensée comme un «can be», la déduction appliquée à un cas concret comme un "actually is» et l'induction comme un "would be». L'induction, en tant que processus progressif de généralisation par lequel nous tendons à la vérité in the long run, correspondrait mieux à la catégorie de tiercéité (Wim Staat, «On Abduction, Deduction, Induction and The Categories», Transactions of C.S. Peirce Society, 1993, 29/2, p. 225-237).

69. M. Berger, «Répondre en citoyen ordinaire», op. cit.

70. Igor Babou, «Randonner avec un vidéo-projecteur. La démocratie participative à l'épreuve dans le parc national de La Réunion», Communication, vol. 34, no 1, 2016. 


\section{Trois modes expressifs}

Considérons à présent la seconde fonction du modèle de Bühler, qui se trouve également triplée dans notre dispositif. Par la fonction expressive, le signe, considéré à présent comme symptôme, renseigne sur l'état psycho-physique du locuteur. Pour les auteurs de la speech act theory comme pour Habermas, l'état du locuteur - «objet» sur lequel les signes de la locution nous donnent plus ou moins de «prise» - peut valider ou invalider les actes de langage qu'il propose.

C'est le cas naturellement pour les actes de langage focalisés sur l'expression, les «actes expressifs» (remercier, s'excuser, congratuler, suppose d'afficher les affects correspondants), mais cela vaut également pour les «actes régulateurs» (ex.: ordre, promesse) et pour les «constatifs» (ex. : simple assertion). La compréhension d'un ordre suppose que soit reconnue comme réelle l'intention de celui qui ordonne; de manière plus évidente, la promesse faite demande au minimum de manifester la sincérité de celui qui s'engage. La compréhension d'une assertion soudaine du type «Il pleut à Sydney» adressée à un ami un jour de beau temps à Bruxelles demande, qu'au-delà de la dimension de vérité du constat, soit reconnu le «ton» et à travers lui les intentions de celui qui cherche à chambrer son ami qui a pour plan de déménager en Australie ${ }^{71}$. Sans cela, si l'énoncé est vrai, l'énonciation n'a pas de sens.

La prétention à la validité expressive d'un acte de langage nous engage comme locuteur à donner à notre interlocuteur des raisons de se fier à notre intention d'établir avec lui le lien illocutoire présenté dans l'acte de langage (gratitude, excuse, ordre, promesse, assertion, etc.), ainsi qu'à notre intention d'en répondre, si l'acte de langage était jugé non valide. Ici, il paraît important d'aller un peu plus loin que Habermas: dans le cas d'actes «engageants», comme les promesses, la validité expressive d'un acte illocutoire ne tient pas seulement à la perception de la sincérité de nos intentions, mais à l'appréciation de la fiabilité de nos intentions éventuellement sincères. La question de la fiabilité du locuteur qui s'engage dans un acte de langage n'intervient pas seulement, comme semble le poser Habermas, à un niveau perlocutoire, comme «suite» réellement donnée à l'acte de langage (par exemple, à la suite d'une promesse, faire réellement ce que l'on a promis). Le jugement de fiabilité intervient immédiatement dans l'illocution, dans la possibilité d'établir communicativement une «binding and bonding relationship ${ }^{72}$ » avec un interlocuteur perçu comme reliable. Autrement dit, la félicité expressive de ces actes de langages ne demande pas seulement de

71. Exemple adapté, à partir de: G. Finlayson, Habermas, Oxford, Oxford University Press, 2005, p. 38.

72. J. Habermas, On The Pragmatics of Communication, op. cit., p. 85-88. 
montrer de la sincérité (to express truthfulness) sur le plan illocutoire et, ensuite, de s'avérer fiable (to prove trustworthy) sur le plan perlocutoire, mais, d'emblée, d'apparaittre fiable quant à nos intentions communicatives.

Une théorie de l'agir communicationnel élargie doit prendre au sérieux cette préoccupation ordinaire des personnes d'apprécier, à partir de signes, la fiabilité des interlocuteurs avec lesquels la communication les lie, et travailler à l'élaboration d'une phénoménologie de la responsabilité d'autrui, qui est d'abord une symptomatologie des états impropres à l'interaction communicative. À cette fin, il faut premièrement reconnaitre que, d'un type de relation illocutoire à l'autre, ces exigences concernant l'état du partenaire varient. Si par exemple des actes de langage de congratulation et de célébration peuvent supporter le fait que celui qui les engage ait bu une dizaine de bières, il en va autrement pour ce qui est de présenter des excuses pour un acte grave.

Il faut ensuite considérer les différents canaux de la communication expressive: ces modes troisièmes, seconds ou premiers que peut prendre le symptôme. Sur ce plan, la théorie de l'expression développée par Erving Goffman à travers son œuvre devrait être reconsidérée par la théorie de l'agir communicationnel, qui l'a rapidement disqualifiée comme une pensée de l'«agir dramaturgique», limitée à sa dimension d'instrumentation stratégique $^{73}$. Certaines distinctions proposées par l'interactionniste américain nous semblent éclairer l'application de la trichotomie peircienne à trois catégories de symptômes.

Des signes peuvent symptomatiser l'état d'un énonciateur sur un mode troisième, c'est-à-dire le «symboliser» : on dira que tel signe exprime "d'habitude», «généralement» ou «typiquement» tel état. Un sourire en coin et une certaine prosodie marquent conventionnellement le sarcasme. Il est possible pour les interlocuteurs de substituer à ces symboles méta-communicationnels un contenu propositionnel du type «il dit le contraire de ce qu'il pense». Le visage fermé, le port de vêtements sombres, les mains croisées devant soi symbolisent ensemble un recueillement attristé, «de circonstance» lors d'un enterrement: un "affect conventionnel», dirait Goffman, ici doublement conventionnel; une première fois quant à la généralité du renvoi du signe à son objet affectif, une seconde fois quant à la généralité de «l'occasion sociale» qui l'exige.

Telle énonciation peut être «symptomatique» sur mode second, lorsqu'un signe - un geste surprenant par exemple - renvoie concrètement vers l'état de la personne, sans qu'on dispose toujours ici de la «représentation média-

73. Daniel Cefaï, Pourquoi se mobilise-t-on? Théories de l'action colletive, Paris, La Découverte, 2007; Mathieu Berger, «L'espace public tel qu'il a lieu», Revue française de science politique, vol. 66, n 1, 2016. 
trice» (signe troisième) nous permettant de reconnaître son état, de «savoir ce qu'elle a», en lui appliquant un contenu propositionnel. Un fait expressif s'est produit, a «frappé nos sens» et a attiré notre attention sur la subjectivité de l'énonciateur, mais le «décodage» de l'expression est problématique, «ce qu'elle veut dire» est incertain ${ }^{74}$. Les symptômes de «type 2» peuvent donc stimuler une enquête à l'échelle de l'interaction, une inspection plus fouillée visant l'émergence de symptômes de «type 3», qui ne se contentent pas d'apparaître de manière saillante, mais «disent» ce qu'ils sont.

Enfin, on peut imaginer qu'un signe symptomatise l'état d'une personne sur un mode premier. Ces symptômes de «type l» sont, de nouveau, plus difficiles à cerner. En nous amenant «au seuil inférieur de la sémiotique», aux marges de la perception, à un niveau infra-indiciel, ils représentent un défi pour l'analyse et pour l'ethnographie de la communication. Pour tout dire, les «phénomènes expressifs» que concernent ces symptômes de type 1 , ne sont plus tout à fait des phénomènes et ne sont plus tout à faits expressifs. Il nous semble en effet pertinent de réserver la notion d'expression aux modes troisième (l'expression comme l'entendait Husserl, comme «vouloir dire», Bedeutung ${ }^{75}$ ) et second (l'expression étant affaire de «pression», de Druck).

Kundgabe, traduit par «manifestation», était la notion initialement choisie par Bühler pour nommer la fonction du langage par laquelle «s'annonce» ou «se donne» la subjectivité des interlocuteurs; il préférera ensuite Ausdruck. L'idée de «manifestation» convient peut-être mieux pour traiter des situations dans lesquelles les signes symptomatiseraient sur un mode premier. On retrouve ici une autre distinction instructive dans les textes de Goffman traitant de l'expression, où il introduit, à côté du verbe to express, le verbe to exude («exsuder»). En deçà d'une «pression» exercée sur les sens, « exsuder» suggère un processus sémiotique diffus, qui opère sur un mode potentiel, comme «pur peut-être» dirait Peirce, plutôt qu'en tant que fait actuel (l'expression surprenante) ou que règle générale (l'expression conventionnelle).

Il est possible de synthétiser ces réflexions relatives aux trois catégories de symptômes à partir de cette trichotomie supplémentaire proposée par Peirce dans le rapport du signe à son objet: Peirce parle de "poti-signes» (signes potentiels, signes en puissance), d'«acti-signes» (signes actuels) et de «famisignes» (signes familiers). Prenons l'exemple des odeurs corporelles dans l'interaction: celles-ci peuvent fonctionner comme des fami-signes, des odeurs

74. Concernant les «regards coupables, les œillades furtives, les hésitations embarrassées», cela renvoie à la différence établie par Ekman entre des «indices de duperie» et des «fuites» plus intelligibles: "Deception clues" tip [the observer] off that deception is in progress but do not reveal the concealed information; the betrayal of that withheld information we call "leakage"» (P. Ekman et F. W. Friesen, cités dans: Erving Goffman, Strategic Interaction, Philadelphie, University of Pennsylvania Press, p. 31n).

75. Jacques Derrida, La voix et le phénomène, Paris, PUF, 1967. 
typiques, générales, par exemple, l'odeur du fumeur. Une odeur âcre qui saisit aux narines et oriente notre attention vers sa source sans pour autant qu'elle puisse être rapportée à un «type» est un acti-signe. Le poti-signe est un signe incertain, simplement possible: pensons au rapport que l'on peut avoir à sa propre haleine: si elle n'est pas particulièrement forte ou marquée (par le café, par la cigarette...), et que nous peinons donc à l'évaluer sensitivement, il est néanmoins possible qu'elle dégage quelque chose et qu'elle renvoie à quelque chose.

\section{Trois modes appellatifs}

Les fonctions cognitive et expressive du modèle de Bühler ont sollicité dans la sémiotique peircienne les différentes modalités du rapport entre un signe et son objet; objet qui pouvait être « externe» dans le cas de la fonction cognitive (les objets et états de choses dans le monde) ou «interne» dans le cas de la fonction expressive (états psychophysiques du locuteur). La dernière fonction du langage, la fonction d' «appel» identifiée par Bühler, par laquelle le signe se fait «signal», demande, pour lui appliquer les catégories peirciennes, de se soucier des différentes modalités du rapport entre un signe et son interprétant. Notons ici, sans avoir le moyen d'approfondir, que «l'interprétant» n'est pas chez Peirce à proprement parler «l'interprète» du signe, mais l'effet qu'appelle le signe.

Parmi les différentes trichotomies proposées par Peirce pour traiter des catégories d'effets du signe, et que nous utiliserons pour qualifier des modes appellatifs différents, il y a celle qui distingue les «rhèmes», les «dicisignes» et les «arguments», et celle qui identifie trois types d'interprétants: "émotionnel», «énergétique» et «logique».

Le rapport du signe à son interprétant, à son effet, est «rhématique» lorsqu'il se présente comme un pure potentialité. Lorsqu'un bébé s'approche en gazouillant «bé-bou-bu», cela peut signifier quelque chose. Le rapport du signe à son interprétant relève du «dicisigne» lorsque le signe «professe», sans plus, son interprétation ${ }^{76}$. En disant distraitement «Désolé...» à un étranger que l'on a bousculé dans une rame de métro, on lui indique qu'il doit comprendre nos mots comme une excuse; on ne lui donne pas d'autres raisons de croire à notre «désolation», et on n'a aucune garantie que ces mots créent en lui l'interprétant «excuse». Enfin, Peirce parle d'«argument» pour qualifier un rapport de nécessité entre un signe et son interprétant. Un argument est un signe qui détermine son interprétant. Nous nous plions nécessairement aux conclusions d'une démonstration mathématique magistrale (pour peu que

76. Thomas L. Short, Peirce's Theory of Signs, Cambridge University Press, 2007, p. 233. 
nous la comprenions suffisamment, sans quoi tout cela resterait pour nous purement rhématique!). Mais nous nous plions également à comprendre et reconnaître des excuses, qui si elles s'accompagnent des manifestations symptomatiques de l'excuse sincère, ont valeur d'argument.

Venons-en à l'autre trichotomie peircienne pertinente pour spécifier la fonction appellative et le signe-comme-signal du modèle de Bühler. L'interprétant logique se passe de longues explications: l'effet créé par le signe se produit par une pensée articulée dans le langage. Ainsi, entendant ma fille gazouiller «bé-bou-bu», je peux avoir compris «j'ai tout bu»: ici, un rhème a produit un interprétant logique. L'interprétant énergétique est traité par Peirce à travers l'exemple de l'ordre militaire «Reposez armes!». Cet ordre de l'officier produit un interprétant énergétique dans le sens que ce qu'il appelle, voire ici, «déclenche», c'est une action. Ici, l'interprétant énergétique est le bruit des armes qui frappent le sol simultanément. Enfin, la musique est évidemment un médium de prédilection pour penser l'interprétant émotionnel: "The performance of a piece of concerted music [...] conveys, and is intended to convey, the composer's musical ideas; but these usually consist merely in a series of feelings ${ }^{77}$ ».

\section{Vers une nouvelle combinatoire}

Ainsi, comme cette dernière citation de C. S. Peirce l'indique, des idées musicales relevant plutôt de la tiercéité peuvent produire un sentiment (feeling), interprétant que Peirce associe à la priméité. L'intérêt d'une spécification du signe-comme-symbole, du signe-comme-symptôme et du signe-commesignal par les trois «catégories» de Peirce tient alors dans la richesse heuristique de la nouvelle combinatoire qu'elle implique. Il est en effet possible d'envisager, sur le plan théorique et dans l'analyse empirique, la multiplicité des sémioses possibles, combinant des symptômes, des symboles et des signaux des trois types ${ }^{78}$. Imaginons différents exemples:

Un symptôme de type 1 , iconique, vague possibilité qualitative liée à l'allure d'un individu «A» dont s'approche «B» dans une rue sombre, peut produire pour «B» un signal de type 2 , énergétique: sans y réfléchir, B change de trottoir. Un symptôme de type 3, basé sur un «stéréotype» (l'apparence de A symbolise pour B un «type» d'individu), pourra produire le même effet. Un individu «C» moins facilement alarmable, en tout cas moins «énergétique-

77. C. S. Peirce, cité dans ibid., p. 203.

78. Et cela, sans même parler de la «spirale infinie de l'interprétation» dans la sémiotique peircienne, par laquelle tout interprétant devient lui-même un signe interprétable à son tour, potentiellement ad infinitum (Umberto Eco, «Unlimited Semeiosis and Drift: Pragmaticism vs. "Pragmatism"», dans Kenneth K. Ketner (dir.), Peirce And Contemporary Thought: Philosophical Inquiries, Fordham University Press, p. 205-221. 
ment» alarmé, pourra lui, face à des signes-symptômes de type 2 pourtant plus factuels et plus saillants (à l'approche de "A», celui-ci pousse un cri ou fait des gestes brusques), traiter le signe sur le mode d'une chaîne d'interprétants logiques («il est seul, il a bu, ce n'est pas à moi qu'il en a, les gens dans ces situations ne sont généralement pas violents ou dangereux») et ne pas dévier physiquement de sa trajectoire. Dans ce dernier exemple décrivant l'attitude de C, l'interprétant logique est un «dicent»: les quelques indices sur lesquels s'appuie $\mathrm{C}$ lui font «se dire que» $\mathrm{A}$ est un homme alcoolisé inoffensif... sans garantie! Le raisonnement est inductif: jusqu'ici, il n'est pas arrivé à C d'être agressé par des hommes ivres.

Prenons à présent la fonction cognitive, la fonction de représentation. Des représentations de type 1 ne produisent pas nécessairement des interprétants de type 1. Un morceau d'ambient music d'un iconisme primaire peut donner lieu à une critique très cérébrale et bavarde (interprétant logique) dans une revue spécialisée. Des représentations de type 3 ne produisent pas nécessairement des interprétants de type 3 . Un propos représentant le monde dans la tiercéité la plus pure pourra susciter un interprétant premier, émotionnel: Peirce parle par exemple de «la qualité de l'émotion éprouvée en contemplant une belle démonstration mathématique ${ }^{79}$ ».

Autre exemple: le jargon de la sémiotique peircienne signifie sur un mode «rhématique» pour ceux qui le découvrent et qui sont peu sensibles à une théorie des signes. On dira "c'est du chinois!», «du charabia!», même si l'on en retiendra bien confusément quelque chose, des significations possibles, un potentiel de signification en attente de codes. L'abstraction de ces signes fera dire à l'un que "ça ne veut rien dire» quand pour un autre une telle abstraction est nécessaire à déterminer les interprétations sémiotiques, à faire «argument» (au sens de Peirce).

La combinatoire de Peirce concernait les 10 classes de signes possibles considérant la combinaison de trois trichotomies: du signe en lui-même, du rapport signe-objet et du rapport signe interprétant. Ainsi, la classe 3-2-2 peut par exemple s'appliquer à un panneau de signalisation officiel (signe de type 3, «légisigne») qui renseigne (rapport à l'interprétant de type 2, « dicent») la direction du centre-ville (rapport à l'objet de type 2 , «indice»).

Transposée dans le modèle de Bühler, la combinatoire, que nous n'avons pu que suggérer à partir d'exemples, peut constituer un instrument de précision pour l'analyse d'interactions communicatives de tous types; des analyses

79. C. S. Peirce, Écrits sur le signe, op. cit., p. 83. 
Figure 3

Une combinaison des trichotomies de Peirce

et les dix classes de signes correspondantes

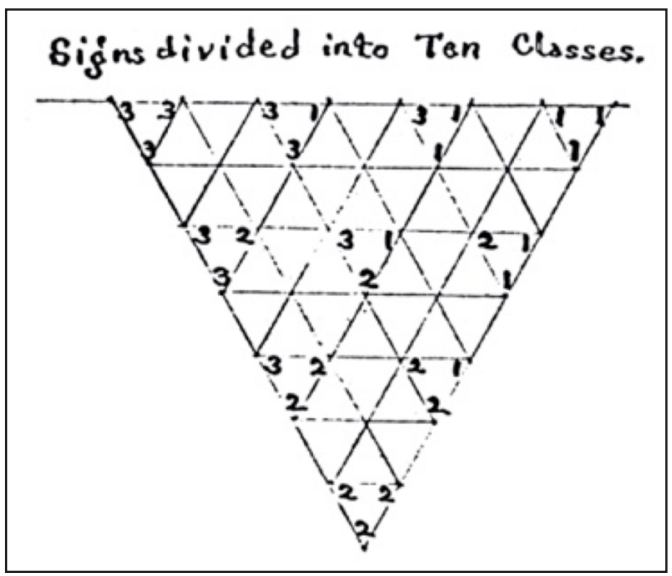

davantage ouvertes au caractère sensible (à la fois «instable», «sensitif» et «sentimental») de la communication, et ce sans rupture avec sa dimension logique, discursive, argumentative. L'idée n'est pas ici de «rompre» avec le rationalisme logocentrique de Habermas pour verser dans la «sensiblerie», mais de travailler dans le sens d'un enrichissement sémiotique de la $T A C$, d'une extension de ce que l'on peut entendre par «rationalité communicationnelle».

\section{Une théorie attentive aux processus symbiotiques et écologiques}

Le troisième auteur mobilisé dans une critique interne visant à sensibiliser la TAC est George Herbert Mead. L'œuvre de Mead est une référence plus centrale encore pour Habermas que celles de Bühler et de Peirce. Dans le second volume de $T A C$, Habermas réfère abondamment à Mead pour théoriser une conception communicationnelle de la rationalité, comme alternative et «thérapie ${ }^{80}$ » face à la rationalisation instrumentale, réifiante, la seule réellement considérée par ses prédécesseurs Adorno et Horkheimer, dans le tableau désespérément sombre qu'ils dressaient de la modernité avancée. Ce faisant, Habermas prolonge, approfondit (puis tente de compléter par une lecture de Durkheim) l'entreprise de Mead de fonder une théorie de la société sur une théorie de la communication: 
[L]es idées de réconciliation et de liberté [esquissées dans la dialectique négative d'Adorno] ont besoin d'être explicitées [...]. Pour ce faire s'impose une théorie de l'action visant, comme chez Mead, le projet d'une communauté de communication idéale. Cette utopie sert en effet à reconstruire une intersubjectivité intacte qui rende possible une communication sans entraves entre les individus ${ }^{81}$.

Une telle «intersubjectivité intacte» n'est rendue possible aux humains que par la disponibilité de cet instrument - permettant à quelqu'un d'entrer en relation avec quelqu'un d'autre à travers la référence à quelque chose ${ }^{82}-$ qu'est $^{\prime}$ le langage. Or, pour Habermas, l'organon nécessaire à la pleine entente et à la production de significations identiques chez les interlocuteurs n'est pas n'importe quel langage : il s'agit du langage du discours, structuré par la grammaire de la différenciation verbale, dont l'usage et la maîtrise n'apparaissent qu'à un certain stade de développement, tant à l'échelle de la vie d'un individu (ontogénèse) qu'à celle de l'évolution de l'espèce (phylogénèse). Pour distinguer la spécificité de l'intercompréhension et de l'entente rendues possibles par «l'interaction régulée par des normes et médiatisée par le langage», Habermas va suivre G. H. Mead dans sa reconstruction d'une «genèse logique» depuis les formes présymboliques, infra-humaines de l'interaction. L'interprétation habermassienne de Mind, Self and Society a connu diverses critiques (Joas, Honneth, Aboulafia, etc.). Dans les limites de l'espace qu'il nous reste, nous tentons quelques éléments critiques qui plaident pour une «mise en situation de la raison» plus décidée, par le biais d'un intérêt théorique accru pour le corps, les gestes et les environnements; pour ce qui, d'un côté, supporte la communication, et de l'autre, la reçoit. C'est sans doute à cette condition qu'une théorie de l'agir communicationnel peut dépasser la conception désincarnée et hors-sol de l'intersubjectivité, et l' «universalisme maigre» reprochés à Habermas ${ }^{83}$.

\section{Les appuis corporels et gestuels de la communication}

En suivant la «genèse logique» proposée par Mead, «depuis l'interaction à ses débuts, alors qu'elle est régie par l'instinct et régulée par des gestes, en passant par l'interaction utilisant le langage des signaux» et des «symboles simples» ${ }^{84}$, le théoricien allemand tend à considérer le langage du «discours avec ses propositions différenciées ${ }^{85}$ » comme une sortie des autres formes de l'interaction, comme le moyen pour la communication humaine de laisser derrière elle l'instinct, le corps, les gestes. En traduisant cela dans les catégo-

81. J. Habermas, TAC 2, p. 8.

82. K. Bühler, Théorie du langage, op. cit, p. 104.

83. M. Aboulafia, "Habermas and Mead...», op. cit.

84. J. Habermas, TAC 2, p. 8 et p. 12.

85. Ibid., p. 8. 
ries de Peirce, les possibilités sémantiques et pragmatiques de sémioses structurées de part en part par la tiercéité (représentation symbolique, expression conventionnelle, interprétant logique), nous libéreraient des limites des communications basées sur des signes iconico-indiciels, et produisant des interprétants infra-logiques. Il en va autrement pour G. H. Mead, chez qui les «gestes», qu'il s'agisse de gestes présymboliques, de significant gestures (ces «symboles simples» non articulés dans une syntaxe) ou de vocal gestures sont essentiels à nos communications, indépendamment de l'émergence d'une compétence discursive.

D’un côté, le corps et les gestes développent leur propre «intelligence» et leur propre potentiel de coordination des interactions entre sujets; un plan de coordination en partie «inconscient» et ne nécessitant pas l'entente sur des «significations identiques» ${ }^{86}$ :

It is not essential that the individuals should give an identical meaning to the particular stimulus in order that each may properly respond. People get into a crowd and move this way, and that way; they adjust themselves to the people coming toward them, as we say, unconsciously. They move in an intelligent fashion with reference to each other, and perhaps all of them think of something entirely different, but they do find in the gestures of others, their attitudes and movements, adequate stimuli for different responses. This illustrates a conversation of gestures in which there is co-operative activity without any symbol that means the same thing to all.

D'un autre côté, le corps et les gestes supportent et accompagnent utilement la communication langagière interpersonnelle, qui, sans eux, "s'effondrerait ${ }^{87} \gg$.

Mais surtout, pour Mead, une énonciation peut elle-même être conçue comme un geste; un geste «vocal» et «significatif». Dans une perspective continuiste, typiquement pragmatiste, le concept meadien de geste traverse les interactions des plus élémentaires aux plus élaborées, des plus «naturelles» aux plus «culturelles». Le geste étant identifié par Mead à "ces commencements d'actes sociaux qui sont des stimuli pour les réponses d'autres organismes ${ }^{88}$ », il peut sans problème s'appliquer aux actes langagiers. Appliqué à l'interlocution, et traduit dans le lexique de Bühler, le geste n'est autre que l'action langagière saisie pour sa «fonction d'appel», sous son aspect de «signal».

\section{Justesse normative et ajustements symbiotiques}

L'énonciation étant conçue, du point de vue du behaviorisme social, avant tout comme stimulus, la fonction de l'appel, le phénomène de réponse, et

86. George H. Mead, Mind, Self and Society, Chicago, The University of Chicago, 1972 (1934), p. 55.

87. Axel Honneth, La société du mépris, Paris, La Découverte, p. 165.

88. George H. Mead, Mind, Self and Society,op. cit., p. 43. 
donc le versant de la réception y ont un statut particulier. Habermas, qui à nouveau s'appuie pour une part importante sur l'œuvre de Mead, rend insuffisamment compte du primat nécessairement donné à la réception par une «pragmatique de la communication» digne de ce nom. Cela apparaît notamment à travers le caractère impropre à nos yeux de la prétention à la validité de «justesse normative» (Richtigkeit) que Habermas propose de faire correspondre à la fonction appellative du langage et donc au pôle de la réception, ainsi qu'à travers le manque de chair et de relief du «monde social», pur univers de normes éthiques ou morales, en référence auquel l'énonciation est appréciée comme valant ou non.

Dans la communication, "l'appel adressé à un autre donne le coup d'envoi» du processus d'intercompréhension ${ }^{89}$. Sans uptake, sans «saisie» de l'énonciation par un récepteur ${ }^{90}$, rien n'a réellement commencé. Il faut pour cela que ce que le locuteur adresse à l'autre le stimule dans un sens apprécié, «lui dise» ou «lui parle» suffisamment. Bien entendu, la «justesse normative» intervient dans ces processus, mais nous ne pouvons y réduire la prétention à la validité liée à la fonction d'appel, ou la condition de félicité du signe-commesignal.

Le problème, tel qu'il nous apparaît, tiendrait au fait que Habermas, dans les exemples convoqués pour établir sa théorie des «prétentions à la validité», mobilise essentiellement des actes de langage régulateurs et en particulier des commandements du genre «Va me chercher un verre d'eau». Pour ces «cas purs d'actes de parole», la question de la justesse normative (l’ordre donné est-il fondé, légitime?) représente certes une condition de félicité évidente et déterminante eu égard à leur capacité d'appel. Non, «ça ne nous dit rien» d'aller chercher un verre d'eau pour un inconnu qui nous l'ordonne, ni même, en tant qu'étudiant, pour un professeur qui croit pouvoir nous le commander. Mais que penser d'une situation dans laquelle le locuteur fait éventuellement preuve d'une justesse normative impeccable sur le plan de l'évaluation éthico-morale de ses propositions, mais irrite et s'aliène immédiatement l'auditeur par la tonalité de sa voix ou son débit de parole? L'infélicité ne tient pas ici au caractère injuste, mais plutôt désajusté, de l'énonciation.

Concernant la fonction d'adresse ou d'appel du langage, une prétention à la validité plus fondamentale, antérieure à la «justesse normative», serait une prétention d'ajustement. Quand je m'engage dans une interaction communicative, je prétends m'adresser à l'autre de façon «appropriée», suffisamment

89. J. M. Ferry, Les grammaires de l'intelligence, op. cit., p. 98.

90. Quels que soient les prélèvements que celui-ci opère par ailleurs, cf. 2.3. 
ajustée à la situation, suffisamment soucieuse des réponses et ajustements de l'autre, qu'ils soient effectifs ou anticipés. Bien que «Richtigkeit» soit parfois traduite comme "appropriateness» ${ }^{91}$, la sémantique juridique de cette prétention à la validité n'honore pas la richesse et la pluralité des ajustements symbiotiques qui, comme le montre Mead, sont génétiquement à l'origine de la communication langagière et continuent de la sous-tendre structurellement.

La question des nécessaires ajustements symbiotiques fait descendre celle de «l'accord» entre les sujets à un niveau infra-discursif, voir infra-symbolique. Le problème est ici d'être suffisamment «accordé» à l'autre et à la situation, au sens musical, que cela concerne la tonalité (attunement) ou le tempo de l'interaction. Ainsi, nombre des désajustements qui produisent les infélicités de la communication tiennent au caractère détonant ou désynchronisé des engagements de parole, par rapport à «ce "comment" dans lequel nous sommes ensemble ${ }^{92} »$. Bien sûr, l'énonciation est susceptible d'être invalidée dans ce cas en tant qu'anomalie, comme comportement «anormal», et il reste possible d'évaluer ces situations à partir de l'idée de «justesse normative». Mais les prises dont nous disposons pour «juger» de celle-ci sont ici essentiellement esthétiques, une affaire de sensations. Elles sont appréciées (appraised), plutôt qu'évaluées (assessed), à travers un interprétant émotionnel ou énergétique une réaction d'agacement par exemple - plutôt que logique.

Habermas, en limitant la dimension esthétique de la communication à la question de l'expression du vécu d'un sujet, et donc à la fonction de manifestation du langage, refuse de manière "étonnamment irréaliste» son importance sur le versant de la réception. Apparait ici la question de la rhétorique, dépréciée par un Habermas hanté par le nazisme, comme «pur instrument de manipulation sociale» et évacuée dans sa $T A C$, en tant que contrainte au libre dialogue. Or on peut se demander, avec H. G. Gadamer, si «toute praxis [n'est pas simplement] inconcevable sans la rhétorique ${ }^{93}$ ", si l'on considère celle-ci au sens plus large que lui donnait Chaïm Perelman. L'aspect rhétorique, appellatif, est inhérent à toute communication, et jusqu'aux formes argumentatives prescrites par Habermas: «quand la figure présente une force argumentative, c'est parce qu'elle souligne et singularise, met en évidence et sensibilise ${ }^{94} \gg$.

91. J. Habermas, On The Pragmatics of Communication, op. cit., p. 77, 80 et 89.

92. M. Heidegger, Être et temps, op. cit., p. 106.

93. Hans-Georg Gadamer, cité dans: Michel Meyer, Principia Rhetorica, Paris, PUF, 2008, p. 62.

94. Michel Meyer, Principia Rhetorica, Paris, PUF, 2008, p. 57. 


\section{Rationalité communicationnelle et rationalité écologique}

Enfin, et en continuité avec ce qui vient d'être dit, dans une théorie établie en référence à Mind, Self and Society, la rationalité communicationnelle ne peut s'élever qu'à partir d'une rationalité écologique, ne peut en tout cas se développer «hors sol», sans contact significatif avec l'environnement ou le milieu qui accueille la communication ${ }^{95}$.

Bien davantage que Habermas, qui subsume la relation de communication intersubjective à des fins d'intercompréhension et de consensus, Mead conçoit celle-ci comme instrument de socialisation ${ }^{96}$. La communication est indissociable, chez Mead, de la community, du tissage de relations de rapprochement, d'intégration, de co-appartenance, etc. Cette communication socialisante (ou aliénante en cas d'échec) n'est pas juste affaire de langage, mais d'engagements pratiques et écologiques, d'involvements, au sens que Goffman a donné à ce terme ${ }^{97}$, mais qui convient aussi pour désigner les jeux d'ajustements chez Mead. Comme l'a posé récemment Daniel Cefaï, la théorie meadienne de l'intersubjectivité et de l'émergence sociale du soi est une écologie. Ce à quoi se socialise l'individu n'est pas une société abstraite mais un «environnement social» concret, situé, et, en multipliant ces processus dans des environnements différenciés, un ensemble de «mondes sociaux ${ }^{98}$ ».

L'exemple donné par Mead pour rendre compte des conversations de gestes de l'homme moderne nous met sur la voie de sa conception de la socialisation: des individus «rejoignent une foule», «s'engagent dans la foule» (get into a crowd); se déplaçant en son sein, ils s'ajustent inconsciemment, mais intelligemment, aux mouvements de ceux qui viennent vers eux; ensemble, ils coopèrent ${ }^{99}$. La socialisation se conçoit à travers des engagements pratiques et dans le mouvement d'une venue à la collectivité ${ }^{100}$, disposée ici en un rassemblement diffus qui reçoit, contient et enclot les processus sociaux qui la composent.

L'émergence de compétences langagières plus élaborées ne change rien à l'orientation fondamentalement socialisante et au caractère fondamentalement gesticulatoire de la communication humaine. Rentrer en communi-

95. D. Shalin, «Critical Theory ans The Pragmatist Challenge», op. cit., p. 254-258.

96. J. Habermas, TAC 2, p. 31-32.

97. Erving Goffman, Behavior in Public Places. Notes on the social organization of gatherings, New York, Free Press, 1963.

98. D. Cefaï, «Mondes sociaux», op. cit.

99. G. H. Mead, Mind, Self and Society, op. cit., p. 55.

100. Pour paraphraser le Venir à la communauté de Stavo-Debauge (Thèse de doctorat en sociologie, Parisn, EHESS, 2009). Voir, à propos des travaux de Joan Stavo-Debauge et leur pertinence pour une sociologie de la communication et de la participation: Mathieu Berger, «La démocratie participative comme épreuve de réception», Sociologies, 2018 (à paraître). 
cation revient à frapper à la porte d'un monde social, à négocier ou reconduire son droit de visite ou d'appartenance. La communication médiatisant toute capacité à accéder à des milieux, à intégrer des groupes et à habiter des mondes sociaux, l'ajustement des gestes ou des symboles adressés représente une condition essentielle à la reconnaissance du sujet et à l'entretien du soi. L'accueil fait à l'énonciation et l'accueil fait à l'individu tendent à correspondre.

Les symboles significatifs et le langage «facilitent» ce processus, en fixant pour le locuteur et son environnement social des significations identiques qui permettent au premier d'envisager et d'anticiper la «configuration de réponses» que produi(rai)t son comportement signifiant auprès d' "un autrui organisé, un autrui généralisé». Se socialiser à un environnement social, c'est, par un effort d'ajustement, accéder à la perspective d'un tel "autrui généralisé», c'est-à-dire «une perspective sur les perspectives» qui composent cet environnement ${ }^{101}$. Cette méta-perspective, à laquelle dans un langage goffmanien correspond le «cadre» de la situation sociale, limite l'incertitude quant à l'indétermination (Bühler) et la multi-modalité (Peirce) de la communication, en fixant un interprétant valant pour les co-participants.

La socialisation, la multiplication des situations et des environnements sociaux auxquel l'individu a affaire étend la compréhension qu'il a d' "autrui généralisé», de ses possibles attentes et réponses, et permet parallèlement «une généralisation progressive du moi» ${ }^{102}$. Dans ses interactions, l'individu mature et autonome peut être amené à dépasser «l'autre organisé» relatif à «une communauté de diamètre restreint» pour en appeler à «une communauté plus élevée qui, en un certain sens, surpasse celle dans laquelle [il se] trouv[e]»103. Par là même, le sujet "projette le contexte d'interaction qui rend possible, à un niveau supérieur, la reconstruction d'une identité [postconventionnelle]» ${ }^{104}$. Si les deux auteurs reconnaissent ce potentiel de transcendance et d'émancipation dans la communication langagière, ils ne lui donnent pas la même extension, et envisagent différemment les rapports entre communauté circonstancielle et communauté plus vaste.

Premièrement, là où Habermas considère de manière fort abstraite les processus sociaux permettant à l'individu de «projeter le contexte d'interaction» dans lequel l'autrui organisé local, étroit, est relativisé par ce qu'on pourrait appeler un autrui universalisé - tout membre d'une «communauté de

101. D. Cefaï, «Mondes sociaux», op. cit.

102. Axel Honneth, La lutte pour la reconnaissance, Paris, Cerf, 2010 (1992), p. 94.

103. G. H. Mead, Mind, Self and Society, op. cit., p. 265.

104. Jürgen Habermas, «Individuation through Socialization: On G. H. Mead's Theory of Subjectivity», dans Postmetaphysical Thinking, Cambridge, MIT Press, 1992, p. 187. 
communication illimitée» ${ }^{105}-$, G. H. Mead approche la socialisation dans des termes qui restent concrets, écologiques, et qui figurent donc des limites à la «vaste communauté» convoquable dans nos communications situées. Comme l'explique Daniel Cefaï, pour Mead, comme pour les sociologues de Chicago qui s'y référeront, la socialisation se conçoit à travers la pluralisation des selves pratiqués dans des situations sociales différentes, et l'extension progressive du réseau des sites et des mondes sociaux auquel l'individu a accès ${ }^{106}$. JeanFrançois Côté parle, lui, d'une «vision kaléidoscopique de la socialité» chez Mead: «c'est véritablement à travers cette myriade d'aspects de la vie sociale qu'apparaît l'existence de la société, dans une réflexion infinie mais néanmoins toujours fragmentaire de sa composition ${ }^{107}$. Cette conception d'un individu développant progressivement ses prises sur une ou des communautés de référence, aussi extensives soient-elles (par exemple, «la communauté scientifique $^{108} \gg$ ) interdit l' 'universalisme mince» qui qualifie l'approche habermassienne de la communication ${ }^{109}$. La seconde différence majeure discutée par Mitchell Aboulafia tient à la déconsidération par Habermas du «moi» (l'aspect objectif, conscientisé et conventionnel du «soi»), par rapport au «je» (l'aspect spontané et créatif), auquel il confie «la tâche d'anticiper la communauté de communication universelle» ${ }^{110}$. Habermas tend à considérer la capacité d'impulsion et de projection du soi attribuée au «je» essentiellement comme possibilité d'arracher l'identité du sujet au «moi» local, qui serait nécessairement «étriqué» (parochial) et «hyper-conventionnel» ${ }^{111}$. Ce faisant, le francfortois dissocie trop abruptement le «je» du «moi» (ces facettes ou «phases» du «soi» sont hypostasiées en entités), ainsi que la "communauté de communication des communautés réelles». Mead cherchait à concilier de manière plus exigeante "universalisme et perspectivisme» ${ }^{112}$, rationalité communicationnelle et rationalité écologique.

La communauté située, les rôles qu'elle organise et le «moi» qu'elle propose doivent être compris et pris en compte par celui qui s'y engage, même (ou surtout?) lorsque ses engagements sont destinés à dépasser ou déplacer les normes du groupe local. Les énonciations du «nouveau venu» et de «l'anormal» - et plus généralement la topique de «l'étranger» ${ }^{113}$

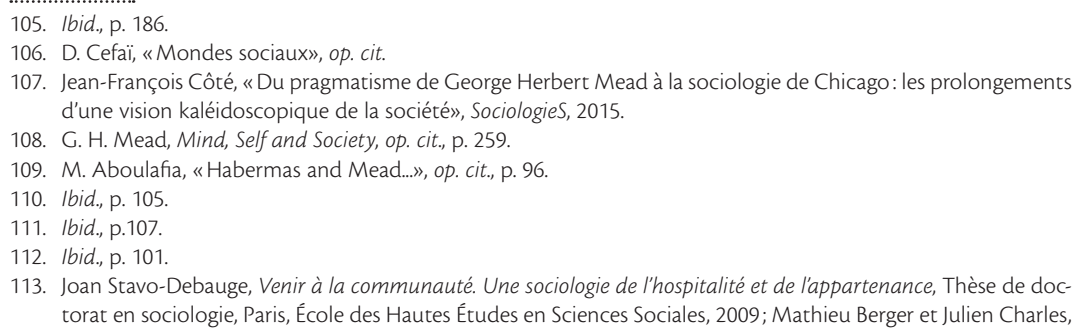

113. Joan Stavo-Debauge, Venir à la communauté. Une sociologie de l'hospitalité et de l'appartenance, Thèse de doctorat en sociologie, Paris, École des Hautes Études en Sciences Sociales, 2009; Mathieu Berger et Julien Charles, 
éclairent ce point. La critique engagée par le nouveau venu au sein d'un groupe, à partir du moment où il ne s'inquiète pas de sa faible socialisation, rencontrera le plus souvent un auditoire impassible, c'est-à-dire indisposé à «pâtir», à recevoir son énonciation. Destinée à subvertir, elle se voit opposer une fin de non recevoir! De même, la «douce folie», l'anormalité assumée et réflexive d'un «personnage haut en couleur» n'est pas celle du «vandale interactionnel» inattentif à sa «place», inconscient de ses «débordements» et des «ravages» qu'ils créent ${ }^{114}$.

Une critique meadienne, interne à la $T A C$, viserait alors à développer le souci théorique pour cette relation à une «communauté vitale» et cette «sensibilité à la structure sociale» (feel for a social structure ${ }^{115}$ ) que parvient ou non à manifester l'énonciateur. À nouveau, nier «l'habitat expérientiel ${ }^{116}$ » de l'interaction langagière, négliger le milieu qui reçoit les énonciations sur le plan écologique, c'est accorder une importance très secondaire à la question de leur réception sur le plan communicationnel; ce qui n'est pas dommageable uniquement sur le plan perlocutoire (comme absence d'effets), mais déjà sur le plan illocutoire de l'entente dans le langage. Ces dernières décennies, Habermas a élaboré une monumentale théorie de l'entente; reste encore à théoriser, au sein d'un même modèle, tout ce que requiert en situation le fait d'être écouté.

\section{Application : une sémiotique de l'excommunication}

La scène se déroule lors d'une séance de Conseil de quartier à Venice Beach, Los Angeles. Je suis assis exactement au milieu d'une salle comptant une centaine de sièges dont la moitié sont occupés. Un homme rejoint le «public» en cours de session et vient s'assoir, une rangée derrière moi et trois sièges sur ma gauche. Trente minutes plus tard, l'homme profitera d'un moment de «commentaires libres du public» pour alerter l'auditoire de l'imminence d'un tsunami dévastateur. D'une voix nerveuse et confuse, l'homme lit difficilement un texte, imprimé sur deux feuilles de papier tirées de sa poche arrière et annonçant la catastrophe pour «ces prochains mois, ou peut-être semaines». Il termine en se félicitant d'être venu prévenir la «communauté de Venice Beach», particulièrement exposée. "It's coming and you're not ready for this. Y'all ain't ready for this. Y'all ain't ready for this!», insiste-t-il, tout en cherchant vraisemblablement dans la salle un regard suffisamment attentif et interpellé. Arrivé à la fin de son temps de parole (deux minutes chronométrées), il est interrompu par le Prési-

«Persona non grata. Au seuil de la participation», Participations, 9, 2014, p. 7-35; Louise Carlier et Mathieu Berger, «Pour une approche pluraliste des figures de l'étranger et des situations-frontières», Sociologies, 2016.

114. Erving Goffman, "The Insanity of Place», Psychiatry, vol. 32, no 4, 1969, p. 357-388. Pour une ethnographie: Mathieu Berger, «Des publics fantomatiques. Participation faible et démophobie», Sociologies, 2015.

115. George H. Mead, Selected Writings, Chicago, The University of Chicago, 1964, p. 404.

116. G. H. Mead, Mind, Self and Society, op. cit., p. 90. 
dent du Conseil, mi-embarrassé, mi-amusé : «Thank you sir.» L’homme regagne son siège un bref instant, puis quitte les lieux.

Nous avons là un exemple d'«infélicité», d'intervention malheureuse, tant sur le plan perlocutoire (rien ne sera entrepris en pratique suite à l'alerte donnée) que sur le plan illocutoire (l'acte d'alerte ne parvient pas à alarmer l'auditoire, si l'on en juge à l'air vaguement amusé ou à l'inattention des destinataires). Importent en particulier dans cette situation, telle que j'en fis l'expérience en tant qu'observateur, certains signes avant-coureurs qui ont soulevé la possibilité, puis m’ont donné la conviction que la prise de parole à suivre serait malheureuse.

Quelques minutes après avoir rejoint son siège à proximité du mien, l'homme, un afro-américain d'une quarantaine d'années, laisse glisser sur le sol l'épaisse veste orange qu'il avait enlevée à son arrivée puis posée sur ses genoux. Il ne la ramasse pas; la veste reste à terre, à ses pieds. Avant de rejoindre la tribune pour son "commentaire", l'homme quitte son siège une première fois pour se rendre au petit buffet installé au fond de la salle. Un verre d'eau et un morceau de cake dans les mains, il regagne sa chaise en marchant sur sa veste au passage. Lorsqu'il quitte une second fois sa chaise, cette fois pour la tribune, en laissant derrière lui la veste orange piétinée, j'ai la conviction que sa prise de parole sera malheureuse. Je ne suis pas particulièrement surpris du thème ni des formes de son intervention, encore moins de la faible réaction de l'auditoire.

Si l'on peut discuter de l'aspect d'icône et d'indice d'un comportement surprenant comme celui de ne pas ramasser pendant de longues minutes un vêtement visiblement tombé au sol et sur lequel on a marché par la suite, à la fois comme analogon (icône) et comme signe annonciateur (indice) d'une prise de parole publique à suivre, il semble plus intéressant de remonter encore dans le processus de formation du trouble et de poser cette question: comment se fait-il, en premier lieu, que j'ai vu cette veste glisser à terre?

L'homme étant assis quelques sièges sur ma gauche mais une rangée en retrait, j'ai dû me retourner légèrement et donc m'orienter vers lui pour voir la veste glisser. Vers la fin de la réunion, une fois l'homme disparu, je réfléchis à la situation et note dans mon téléphone: "Quelque chose me dispose à voir sa veste tomber; quelque chose d'étrange pouvait venir de lui.» Ici, on peut penser que c'est en tant que catégorie sociale ou phénotype - c'est-à-dire sur le plan symbolique et en toute tiercéité - que cet individu m'apparaît, a mon attention, suscite des attentes et des anticipations. Or l'individu n'apparaît pas particulièrement «marginal» et ce n'est pas non plus en tant que Noir qu'il attire mon attention (il y a autour de moi de nombreux autres Afroaméricains). 
Je voudrais défendre l'idée que les signes qui commencent à me disposer d'une certaine manière vis-à-vis de cet individu et initialisent une sémiose de la bizarrerie ne sont ni symboliques, ni indiciels, mais iconiques, ces «symptômes de type l», si difficilement saisissables et qui nous amènent "au seuil inférieur de la sémiotique», dirait Umberto $\mathrm{Eco}$. $\mathrm{Si}$, dès son arrivée, je suis sensibilisé à la présence de cet invididu, ce n'est pas pour ce qu'il représente symboliquement, ni pour ce qu'il présente ostensiblement commme signes d'étrangeté. Ce n'est pas parce qu'il affiche un «type», ni parce qu'il transgresse des «normes» ou des «conventions» ${ }^{117}$; ni en raison de signes visuels, auditifs, olfactifs qui auraient «frappé mes sens» et «forcé» mon attention. Sont plutôt en cause ici de subtils désajustements symbiotiques (misattunements) dans l'ordre de l'interaction, occasionnant une tension interactionnelle d'un niveau très bas: l'arrivée de cet homme, sa présence, une rangée en retrait et trois sièges sur ma gauche, ont produit une légère différence dans la tonalité affective de la situation, dans l'ambiance: «l'être en commun $[\ldots]$ est autre, il a changé de ton» ${ }^{118}$.

Il est possible que sa fébrilité, qui se montrera puis se démontrera par la suite, m'était déjà accessible dès son arrivée, à la limite de mon champ perceptif et à la «marge de ma conscience» ${ }^{119}$. À ce stade, si je suis vaguement conscient de la présence de l'homme mais que mon attention ne s'y arrête pas, on peut dire que sa fébrilité signifie sur un mode infra-indiciel, pré-indiciel. Elle n'est pas encore un indice, tant sur le plan qualitatif (rien de criant, de saillant, ne se produit à ce stade), que temporel (elle me prépare à me montrer attentif au surgissement d'indices). À nouveau, cette distinction entre symptôme de type 2 et symptôme de type 1 me semble renvoyer à la différence que Goffman envisage, mais ne développe pas, entre ce qu'un comportement «exprime» (qui comprend l'idée d'une «pression», Druck, relevant de la secondéité) et ce qu'une présence «exsude», ce qui en «transpire». Ce mode premier du symptôme correspond au mode premier de la cognition que nous évoquions dans le cas de l'ambiance morose d'un espace urbain : par la modification qu'elle introduit dans le «ton» de l'«être en commun», la présence de «l'homme attristé» de Heidegger, ou, ici, la manifestation diffuse de la bizarrerie, a des qualités atmosphériques, elle génère autour d'elle une ambiance. Autrement dit, et c'est le sens même de cet exemple,

117. Joan Stavo-Debauge, «Sous les catégorisations: de l'allure du nouveau venu aux figurations de la culture de l'étranger», Louvain-La-Neuve, CriDIS Working Papers, 2017, no 67. Notre attention pour ces situations et ce que nous appelons ici «symptômes de type 1» doit d'ailleurs beaucoup au concept d'«allure» tel que StavoDebauge le développe, et aux descriptions qu'il consacrait à «l'allure du nouveau venu» dans sa thèse de doctorat (J. Stavo-Debauge, Venir à la communauté, op. cit., p. 576-626).

118. M. Heidegger, Être et temps, op. cit., p. 106.

119. Aaron Gurwitsch, Théorie du champ de la conscience, Paris, Desclée De Brouwer, 1957. 
la question de l'exclusion ou plus précisément de «l'excommunication» ${ }^{120}$, la disposition de la communauté des communicants à tracer ses limites et à se détourner des indésirables ${ }^{121}$, peut intervenir très tôt, à niveau très bas, que ce soit sur le plan du renvoi du signe à son objet (le signe n'est qu'un "poti-signe», un signe potentiel), ou sur celui de l'interprétant produit (un interprétant «émotionnel» de malaise ou «énergétique» de répulsion).

On comprend évidemment aussi comment ces situations peuvent en appeler à ce qu'il y a de plus bas en nous dans notre rapport d'aversion visà-vis de certains inconnus; à ces affects anti-démocratiques sur lesquels a insisté Martha Nussbaum ${ }^{122}$. Ceux-ci affectent, notamment, mon attention d'observateur-ethnographe de cette scène. Quelques mois plus tard, alors que je présentais cette situation dans un colloque, une collègue demande: «Est-ce que c'était vraiment n'importe quoi son intervention à propos du tsunami? Après tout, il se trouve à Los Angeles, et ils attendent là-bas des tremblements de terre importants pour bientôt... Il pouvait avoir des arguments, non?» J'étais incapable de lui répondre. Observateur «attentif» de la scène, j'ai finalement porté peu d'attention aux contenus de l'intervention de cet homme, à ce qui se trouvait inscrit sur les feuilles de papier chiffonnées sorties de sa poche et qu'il lisait péniblement à la tribune. Nous retrouvons ici la théorie des aspects du signe de Bühler: impassible vis-à-vis de ses efforts à «représenter» des états de choses, mon attention portait sur ce qu'il «manifestait»; sur son état à lui. Le signe-comme-symbole s'effaçait devant le signe-comme-symptôme.

Enfin, il est évident que sur le moment je ne suis pas le seul à appréhender cette intervention sous son aspect symptomatique. Autour de moi, les personnes présentes ne sont pas alarmées par cette alerte au tsunami. La réaction commune qui me parvient est celle de visages mi-embarrassés, miamusés; celle d'une communauté située, partageant un certain nombre d'attentes quant aux prises de parole citoyennes dans un Conseil de quartier à Venice, et n'entretenant généralement pas d'espoirs importants quant à ce qui peut se dire et s'entendre en seconde partie de réunion, dans ces séquences consacrées aux «divers», aux «commentaires libres du public». L'intérêt écologique - auquel invite l'approche meadienne - pour l'«habitat expérientiel» de l'interaction langagière demande à l'enquêteur d'être attentif à l'organisation d'un auditoire et aux «réponses communes» qu'il

120. «Improper conduct in one situation can bespeak a general disenfranchisement in face-to-face interaction. Such conduct need not arise from a psycho-pathological condition; presumably it can, however, give rise to one through the response the individual may make to his excommunication» (Goffman, Behavior..., op. cit., p. 216).

121. M. Berger et J. Charles, «Persona non grata», op. cit.

122. Martha Nussbaum, Les émotions démocratiques, Paris, Flammarion, 2011. 
produit. Cette (im)passibilité communicationnelle qui nous a intéressé dans ce texte et sur laquelle le sociologue de la discussion publique doit se pencher ne concerne en effet pas tel auditeur individuel ou tel autre; elle a une dimension collective et qualifie plus largement des milieux de réception dont nous devrions mieux comprendre et expliquer l'attention sélective. 